\title{
Genome-wide bioinformatics analysis revealed putative substrate specificities of SABATH and MES family members in silver birch (Betula pen- dula)
}

\author{
Kiran Singewar ${ }^{1,2}$, Christian R. Moschner ${ }^{1}$, Eberhard Hartung ${ }^{1}$, Matthias Fladung ${ }^{2}$
}

\begin{abstract}
'Institute of Agricultural Process Engineering, Christian-Albrechts University of Kiel, Max-Eyth-Str. 6, 24118 Kiel, SchleswigHolstein, Germany.

${ }^{2}$ Thünen-Institute of Forest Genetics, Sieker Landstraße 2, 22927 Grosshansdorf, Schleswig-Holstein, Germany.
\end{abstract}

*Corresponding author: Matthias Fladung, E-mail: matthias.fladung@thuenen.de

\begin{abstract}
Plant SABATH family members catalyze the methylation of many hormones, signaling molecules, and floral scent metabolites, including salicylic acid (SA), jasmonic acid (JA), and indol-3 acetic acid (IAA). Demethylation of resulting methyl esters was executed by members of the MES family. Members of both families are significantly involved in plant developmental processes. Here, using different bioinformatics tools, we studied the evolutionary relationship and characterized the putative functions of the family members in silver birch (Betula pendula). It is a socio-ecologically important tree species and plays a vital role in reforestation. Ten and twelve members of the SABATH (BPSABATH1-10) and MES (BpMES1-12) family were identified in silver birch, respectively at the gene and enzyme levels. The BpSABATH and BpMES genes were distributed on seven of fourteen chromosomes, indicating the occurrence of moderate duplication events important for the expansion of both families. Phylogenetic clustering and the gene ontology database suggest, BpSABATH8 is involved in the methylation of indole-3-acetic acid (IAA), while BpSABATH5, BpSABATH6, and BpSABATH7 methylate JA to methyl jasmonate (MeJA). BpSABATH9 was alone in the phylogenetic functional group 1 and prefers $S A$ as a substrate to synthesize methyl salicylate (MeSA). Likewise, BPMES5 and BpMES12 are possibly involved in the demethylation of the methyl ester of IAA, while BpMES6, BPMES7, and BpMES8 are responsible for the demethylation of MeJA. BpMES9 clustered with MES and prefers MeSA as a substrate. The current analysis helped to select candidate genes that could be subjected to further molecular breeding of birch varieties adapted to biotic and abiotic stress conditions.
\end{abstract}

Keywords: Birch; Bioinformatics, SABATH; MES; Phylogeny; Substrate prediction

\section{Introduction}

Many plant metabolites, including salicylic acid (SA), jasmonic acid (JA), and indol-3 acetic acid (IAA), undergo methylation and demethylation in different environmental conditions (D'Auria et al., 2003, Han et al., 2017, Yang et al., 2008). The methylation of these metabolites is catalyzed by the members of SABATH enzyme family, a group of S-adenosyl-L-methionine (SAM)-dependent methyltransferases (SAM-MTs) representing an associated group of $O$-methyltransferases (OMTs) (D'Auria et al., 2003). The intra- and inter-specific comparative analysis showed high sequence similarities in the SABATH family members, though the individual members express different substrate specificities (Han et al., 2017, Yang et al., 2008).

The SAM: salicylic acid carboxyl MT (SAMT) and benzoic acid carboxyl MT (BAMT) from Clarkia breweri and Antirrhinum majus, respectively, were the first two enzymes isolated and characterized from the SABATH family (Dudareva et al., 2000, Ross et al., 1999). The cofactor SAM is the most widely used methyl donor for enzymatic methyl transfer (Joshi et al., 1998). The name SABATH was designated based on the first three identified and characterized genes (SAMT, BAMT, and Theobromine synthase). Although different members of the SABATH gene family showed high nucleotide sequence similarities with many plant species, their numbers vary considerably. In Arabidopsis thaliana (AtSABATH), and Oryza sativa (OsSABATH), a total number of 24 , and $41 S A B A T H$ genes, respectively, were 
identified. The crystal structure of the $A$. thaliana IAA methyltransferase (AtIAMT) was determined and the OsSABATH4 gene was identified as the most similar to AtIAMT. More than half of OsSABATH genes were expressed in leaves, roots, and stems representing their active participation in diverse molecular processes (Zhao et al., 2008). A variety of plant mechanisms are responsible for regulating the methylated and free forms of IAA (Delker et al., 2008, Teale et al., 2006). In A. thaliana and Populus trichocarpa, MelAMT catalyzes the methylation of IAA (Zhao et al., 2008) involved in leaf development (Qin et al., 2005). The woody plant species Picea abies (PaSABATH1-10), Picea glauca (PgSABATH1-15), and P. trichocarpa (PtSABATH1-28) contain 10, 15, and 28 enzyme family members, respectively (Chaiprasongsuk et al., 2018, Han et al., 2017, Zhao et al., 2009). The enzymatic activity of the ten PaSABATHs was tested against IAA, SA, and JA phytohormones. The higher enzymatic activity with IAA and SA was shown by PaSABATH 1 and PaSABATH2, respectively, while three PaSABATHs (4, 5, and 10) elected JA as a substrate (Chaiprasongsuk et al., 2018). Further, comprehensive evolutionary and biochemical functional analysis disclosed the change in substrate specificity upon a shift in a single amino acid in forward and reverse mutagenesis studies (Han et al., 2017). The finding indicates the fine-tuned regulation of the SABATH enzyme family members in woody plant species.

The demethylation of the resulting methyl esters of SA (MeSA), JA (MeJA), and IAA (MelAA) is catalyzed by the members of the methylesterase (MES) enzymes, which is affiliated to the $\alpha / \beta$ hydrolase superfamily (Nardini et al., 1999). The first MES, salicylic-acid binding protein 2 (SABP2), was isolated from Nicotiana tobacco and was studied in the SA signaling pathway (Kumar et al., 2003). The amino acid sequence of NtSABP2 shares $77 \%, 46 \%$, and $56 \%$ similarity with P. trichocarpa MeSA, Solanum lycopersicum MeJA, and Rauvolfia serpentina polyneuridine aldehyde esterase (PNAE), respectively (Dogru et al., 2000, Stuhlfelder et al., 2004, Yang et al., 2008, Zhao et al., 2009). In only two species, A. thaliana (AtMES) and the Vitis vinifera (VvMES), a total number of 20 and 15 members, respectively, of the MES gene family were identified (Yang et al., 2008, Zhao et al., 2016). Of the three members that showed enzymatic activity towards MeJA, VvMES5 was $77 \%$ identical to $S$. lycopersicum MeJA at the protein level (Zhao et al., 2016). Further, the VVMES5 denoted as VVMJE1 and its differential expression was evaluated with heat, cold, and UV-B-treated $V$. vinifera plants. Upregulation (in the expression) of VVMES1 upon cold and UV-B treatment was observed, suggesting its role in response to abiotic stresses. The active participation of MeJA in keeping fruits and vegetables fresh has also been demonstrated (Alvarez et al., 2015).

Biochemical analysis revealed, AtMES17 (At3g10870) vigorously catalyzes the hydrolysis of methyl ester IAA (MeIAA). However, AtMES17 with T-DNA insertional mutant lines resulted in reduced sensitivity to MelAA in comparison to wild-type roots of $A$. thaliana plants. In the same study, A. thaliana plants overexpressing AtMES17 showed induced activity to MelAA and not to IAA. The study also suggests that AtMES17 participates in IAA homeostasis and the MeIAA is not a form of an active auxin. It could be possible that the auxin is transported in the form of MelAA due to its better nonpolar nature than IAA. (Yang et al., 2008). A recent study with Citrus sinensis has shown the participation of CsMES in the hydrolysis of MeSA into SA through molecular modeling. It could be demonstrated that the citrus canker caused by Xanthomonas citri is suppressed by SA and MeSA (Lima Silva et al., 2019). Accumulation of SA and CsMES occurred in the course of $X$. aurantifolii and $C$. sinensis interaction. The finding advocates the role of MeSA and $S A$ in the pathogen-induced systemic acquired resistance (SAR) mechanism.

The role of SABATH and MES enzyme families in the synthesis of hormones, signaling molecules, and floral scent metabolites necessary for plant development have been mostly studied in the model and crop plants (D'Auria et al., 2003, Yang et al., 2008). Thus, the information about these enzymes in the woody plant species is very limited. Considering the importance of forest trees for the ecosystem in the present era of climate change, it is advantageous to study the SABATH and MES enzymes in long-lived woody plant species. Here, B. pendula (silver birch) was selected for the in-silico analysis since it is one of the dominant species in the boreal forests of the Northern Hemisphere (Salojärvi et al., 2017). Silver birch is a commercially important tree species and plays a vital role in landscape structure, forestry, breeding for biomass production, and horticulture (Ashburner et al., 2013). The leaf color of birch transforms to yellow-green in autumn and is usually green in the springtime and summer (Gang et al., 2019). The characteristics like short life cycle, rapid growth and plentiful production of seeds make birch a pioneer species that participate in the regeneration of forests after 'forest fires' (Fischer et al., 2002). Different species of the genus Betula, adapting to various climatic conditions, are distributed within a wide geographical region (Hemery et al., 2010, Hynynen et al., 2009). Birch is a wind-pollinated species, widely involved in cross-pollination (Atkinson 1992, Koski et al., 2005) and creating a large gene pool (Ranta et al., 2008). Thus, high genetic variability is maintained giving rise to tolerance formation and increasing the probability of survival in diverse environmental conditions (Araminienè et al., 2014, Aspelmeier et al., 2004). Birches create ideal living conditions for other tree species (Prévosto et al., 2004, Rosenvald et al., 2014), and thus, they significantly contribute to the recovery of forests after disturbances (Dubois et al., 2020). Silver birch plays a key role in maintaining the biodiversity of coniferous forests since the species coexists with other tree species (Hynynen et al., 2009).

The present study aims to (1) identify and characterize the SABATH and MES genes in B. pendula; (2) facilitate our understanding of the evolution and the putative substrate specifications of SABATH and MES enzyme members; and (3) provide useful bioinformatics information for the selection of appropriate candidate genes involved in the methylation and demethylation of SA, JA, and IAA in B. pendula.

To this end, we have successfully characterized the gene and enzyme members of the two SABATH and MES families in $B$. pendula by in silico analyses. The different bioinformatic analyses were crucial and assisted in designing further state-of-art 
molecular and biochemical experiments to evaluate their functional role in B. pendula.

\section{Materials and Methods}

\section{Identification of SABATH and MES gene family members in B. pendula}

The amino acid sequences of Clarkia breweri SAMT (CDSAMT) and $N$. tabacum SABP2 (NtSABP2) were obtained from previous studies (Kumar et al., 2003, Ross et al., 1999). The amino acid sequences of the two genes were used as queries in a tBLASTn search of the $B$. pendula genome sequence (Bp). An E-value cutoff of $1^{-5}$ was applied to the homolog recognition and if the sequence satisfied the criteria, it was selected as a candidate gene.

\section{SABATH and MES amino acid sequence retrieval from different plant species}

Only functionally characterized members from $A$. thaliana (AtSABATH), P. trichocarpa (PtSABATH) and P. abies (PaSABATH) (Chaiprasongsuk et al., 2018, D'Auria et al., 2003, Zhao et al., 2013), A. thaliana (AtMES), V. vinifera (VvMES) (Yang et al., 2008, Zhao et al., 2016), and other known members from different species were retrieved from NCBI (https://www.ncbi.nlm.nih. gov/) and Popgenie (http://popgenie.org/) databases for the comparative analysis (S Table 1).

\section{Multiple sequence alignment and phylogenetic analysis}

The retrieved amino acid sequences were aligned using the ClustalW program (Thompson et al., 1994) available in the MEGA X bioinformatics package (Kumar et al., 2018) with default parameters. The maximum likelihood $(M L)$ gene trees of BpSABATH and BpMES with other known SABATH and MES enzymes were constructed using 1000 bootstrap replicates in the MEGA X bioinformatics tool (Kumar et al., 2018). Aspergillus niger SAMT (NT166520) and Beauveri abassiana MES (PMB68924.1) were used as outgroup species in the construction of the gene tree for substrate prediction.

\section{Chromosomal localization of birch SABATH and MES genes}

A physical map was drawn to confirm the chromosomal locations of the SABATH and MES genes. The karyoploteR (Gel et al., 2017) package was used to plot the chromosome map and to visualize the locations of SABATH genes on the B. pendula chromosomes.

Tandem duplications in the BPSABATH and BPMES gene family were determined when located within $100 \mathrm{~kb}$ neighboring regions and when a close phylogenetic relationship was formed among a group of genes at the same chromosome location (Kong et al., 2007).

\section{Gene structure, conserved domain, gene ontology, and promoter analysis}

Coding regions (CDSs) and genomic sequences were retrieved from the B. pendula genome (https://genomevolution.org/ coge/Genomelnfo.pl?gid=35080 to analyze the intron/exon organization of BPSABATH and BPMES genes. Further, the sequences were submitted to the Gene Structure Display Server (http://gsds.cbi.pku.edu.cn/) to investigate the gene structure based on each of the CDSs and the corresponding genomic sequences.

The MEME online tool (http://meme-suite.org/) was utilized to identify the motifs present in the BPSABATH and BPMES genes. The following parameters were set: the maximum number of motifs, 11; minimum motif width, 6; maximum motif width, 60 . Additionally, all predicted SABATH and MES gene family members in $B$. pendula were submitted to the Pfam database (El-Gebali et al., 2018) to confirm the conserved domains of all candidate genes (https://www.genome.jp/ tools/motif/).

The theoretical isoelectric point $(p l)$ and molecular weights $(M w)$ of the SABATH and MES enzymes in B. pendula were predicted using the 'Compute $\mathrm{pl} / \mathrm{Mw}$ tool' on the ExPASy server (https://web.expasy.org/compute pi/). Besides, the promoter regions of BPSABATH and MES genes were examined in the PlantCARE database (Lescot et al., 2002). DNA fragments of approximately $1,000 \mathrm{bp}$ were retrieved from the 5 '-untranslated region of the genes. Further, the raw sequences were subjected to the PlantCARE database and the option 'search for care' used to search for Cis-regulatory elements.

The $B$. pendula gene ontology browser available at the Hardwood Genome project (https://www.hardwoodgenomics. org/organism/Betula/pendula) was used to attribute the product of the BPSABATH and BpMES genes. Further, the protein structure homology-modeling of all the family members was carried out to validate the functional residues. The SwissModel, an automated server (https://swissmodel.expasy.org/), was used to build the protein models.

\section{Results}

\section{Identification and comparative analysis of SABATH and MES genes}

To identify the SABATH and MES gene family members in B. pendula, BLASTP analyses against the $B$. pendula genome were performed using amino acid sequences of CDSAMT for SABATH and NtSABP2 for MES as queries. A total number of 10 and 12 SABATH and MES most similar genes were obtained (Table 1). Protein sequences of both family members were subjected to Pfam analyses to confirm their protein domain.

Gene lengths of BPSABATH varied from 1,730 (BPSABATH10: Bpev01.c0800.g0038.m0001) to 21,489 bp (BpSABATH9: Bpev01.c0425.g0055.m0001). The lengths of the BpSABATH CDS and protein varied from $453 \mathrm{bp}$ and 150 aa (BpSABATH1: Bpev01.c2345.g0001.m0001) to 1,548 bp and 
515 aa (BpSABATH5: Bpev01.c0161.g0056.m0001), respec-

tively (S Table 2).

Table 1

The SABATH and MES family members in B. pendula. A total number of 10 and 12 members were obtained, respectively. The gene locus, chromosome number, length of nucleotide sequence, protein, and CDS sequences were determined using the $B$. pendula genome.

\begin{tabular}{|c|c|c|c|c|c|}
\hline BpSABATH and BpMES & Gene locus & Chromosome & Nucleotide sequence (bp) & CDS (bp) & Amino Acid (aa) \\
\hline BpSABATH1 & Bpev01.c2345.g0001.m0001 & Chr1 & 5,177 & 453 & 150 \\
\hline BpSABATH2 & Bpev01.c1865.g0002.m0001 & Chr1 & 2,113 & 858 & 285 \\
\hline BpSABATH3 & Bpev01.c0759.g0006.m0002 & Chr1 & 3,566 & 966 & 321 \\
\hline BpSABATH4 & Bpev01.c0807.g0007.m0001 & Chr3 & 9,210 & 954 & 317 \\
\hline BpSABATH5 & Bpev01.c0161.g0056.m0001 & Chr9 & 5,154 & 1,548 & 515 \\
\hline BpSABATH6 & Bpev01.c0161.g0057.m0001 & Chr9 & 2,693 & 1,125 & 374 \\
\hline BpSABATH7 & Bpev01.c0161.g0058.m0001 & Chr9 & 2,793 & 1,107 & 368 \\
\hline BpSABATH8 & Bpev01.c0240.g0011.m0001 & Chr12 & 3,226 & 1,029 & 342 \\
\hline BpSABATH9 & Bpev01.c0425.g0055.m0001 & Chr12 & 21,489 & 1,344 & 447 \\
\hline BpSABATH10 & Bpev01.c0800.g0038.m0001 & Chr13 & 1,730 & 1,020 & 340 \\
\hline BpMES1 & Bpev01.c0449.g0051.m0001 & Chr1 & 4,323 & 1,164 & 258 \\
\hline BpMES2 & Bpev01.c0919.g0029.m0001 & Chr1 & 15,239 & 996 & 214 \\
\hline BpMES3 & Bpev01.c0135.g0098.m0001 & Chr2 & 5,954 & 1,140 & 260 \\
\hline BpMES4 & Bpev01.c0436.g0011.m0001 & Chr3 & 1,764 & 738 & 167 \\
\hline BpMES5 & Bpev01.c1072.g0010.m0001 & Chr5 & 2,691 & 813 & 195 \\
\hline BpMES6 & Bpev01.c0015.g0216.m0001 & Chr5 & 1,014 & 765 & 170 \\
\hline BpMES7 & Bpev01.c0015.g0217.m0001 & Chr5 & 2,819 & 783 & 170 \\
\hline BpMES8 & Bpev01.c0015.g0218.m0001 & Chr5 & 3,644 & 780 & 172 \\
\hline BpMES9 & Bpev01.c0015.g0219.m0001 & Chr5 & 2,099 & 792 & 180 \\
\hline BpMES10 & Bpev01.c0015.g0220.m0001 & Chr5 & 5,425 & 561 & 121 \\
\hline BpMES11 & Bpev01.c0015.g0221.m0001 & Chr5 & 1,612 & 627 & 147 \\
\hline BpMES12 & Bpev01.c0089.g0060.m0001 & Chr13 & 6,019 & 777 & 179 \\
\hline
\end{tabular}

The gene lengths of BpMES varied from 1,014 bp (BpMES6: Bpev01.c0015.g0216.m0001) to 15,239 bp (BPMES2: Bpev01. c0919.g0029.m0001). The lengths of the BpMES proteins and CDSs varied from 121 aa to 260 aa (BpMES10: Bpev01.c0015. g0220.m0001 and BpMES1: Bpev01.c0449.g0051.m0001) and $561 \mathrm{bp}$ to 1,164 bp (BpMES1: Bpev01.c0449.g0051.m0001 and BpMES10Bpev01.c0015.g0220.m0001), respectively (S Table 2).

The molecular weights of the predicted BpSABATH enzymes ranged from $16.90 \mathrm{kDa}$ (BpSABATH1) to $48.67 \mathrm{kDa}$ (BpSABATH9), and the theoretical isoelectric points were predicted to range from 4.91 (BpSABATH1) to 8.75 (BpSABATH9) (S Table 3). The molecular weights of BpMES enzymes ranged from $22.01 \mathrm{kDa}$ (BpMES10) to $42.68 \mathrm{kDa}$ (BpMES1), and the theoretical isoelectric points were predicted to range from 5.20 (BpMES6) to 9.41 (BpMES11) (S Table 3).

\section{Chromosomal localization of B. pendula SABATH and MES genes}

Analysis of the chromosomal locations showed that the ten and twelve SABATH and MES genes each mapped to only five chromosomes and were unevenly distributed throughout the genome (Figure 1). Of the 10 BpSABATH genes, three were located on chromosomes 1 (BpSABATH1, BpSABATH2, and BpSABATH3) and 9 (BpSABATH5, BpSABATH6, and BpSABATH7), with two genes on chromosome 12 (BpSABATH8 and BpSA$B A T H 9)$. In contrast, the two genes BPSABATH4 and BPSABATH10 were located on chromosomes 3 and 13, respectively.

Of the 12 BpMES genes, only one gene each was located on chromosomes 2 (BpMES3), 3 (BpMES4), and 13 (BpMES12), with two genes located on chromosome 1 (BPMES1 and BPMES2). In contrast, seven BPMES genes (BPMES5, BpMES6, BPMES7, BPMES8, BPMES9, BPMES10, and BpMES11) were located on chromosome 5.

Considering that duplication events are more likely to be customary in the gene family expansion (Moore et al., 2003), the possibilities of tandem and segmental duplications of the $B p S A B A T H$ and BpMES genes were investigated (Figure 1). Of the $10 B p S A B A T H$ genes, five were found in two tandem repeats, including BPSABATH1 with BPSABATH2, and BPSA$B A T H 5$ and BpSABATH6 with BpSABATH7. Eight of the 12 BpMES genes were found in two tandem repeats, including BpMES1 with BpMES2, and BPMES5, BpMES6, BpMES7, BPMES8, BpMES9 and BPMES10 with BPMES11. 

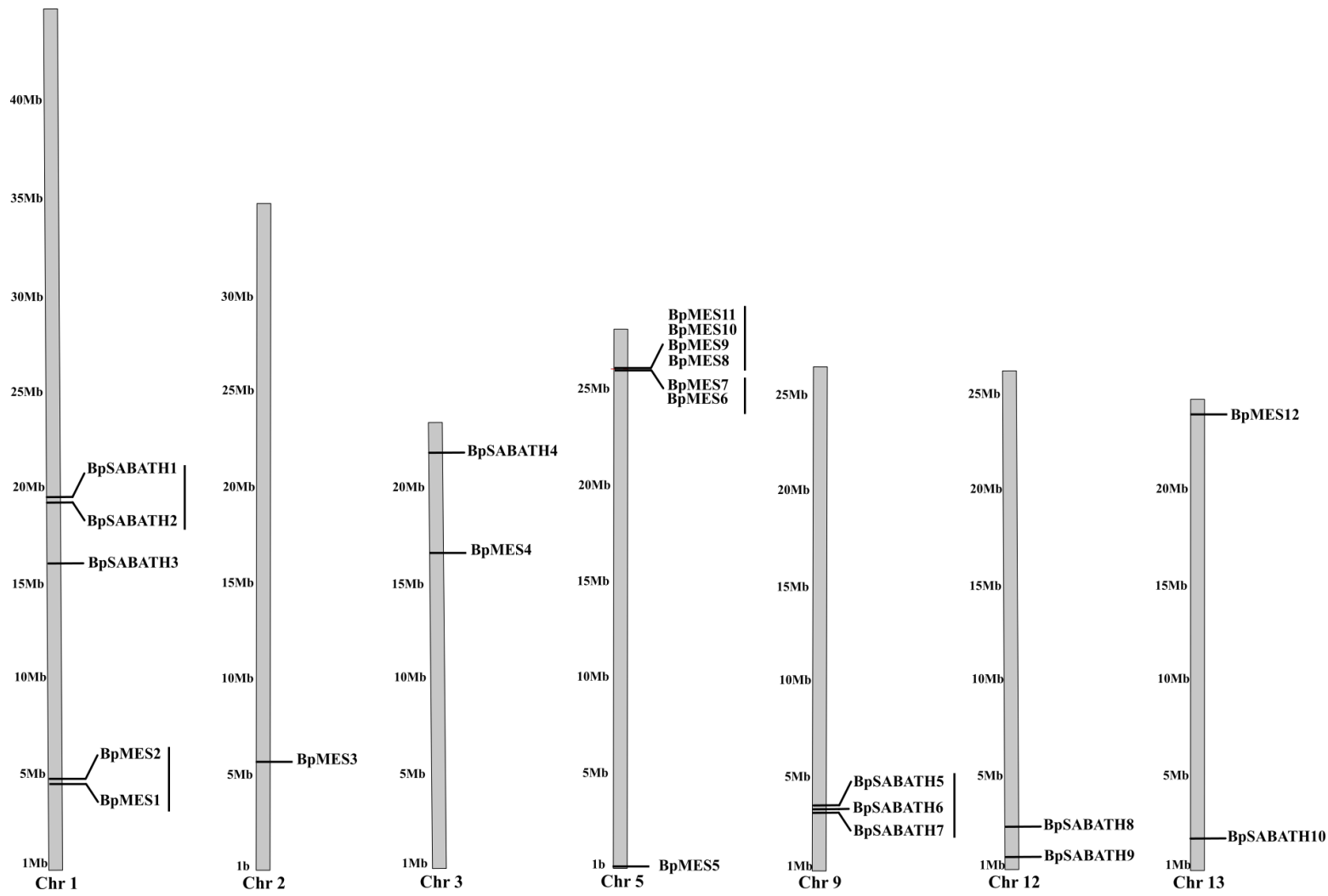

Figure 1

Localization of SABATH and MES genes on silver birch (B. pendula) chromosomes: Chromosome 1 and 9 carry three SABATHs each, while chromosome 3 and 13 carry only one SABATH gene. Chromosome 2 and 5 carry one and seven MES genes, respectively. Chromosome 1, 3, and 13 contain both SABATH and MES genes. The names of the chromosomes and their sizes $(\mathrm{Mb})$ are indicated next to each chromosome and are based on the $B$. pendula genome. Tandemly duplicated genes are shown beside the black lines. No evidence of segmental duplication was identified in the SABATH gene family in the B. pendula genome. The karyoploteR package was used to plot the chromosome map.

\section{Gene structure and intraspecies phylogenetic relationship analysis (gene tree) of BpSABATH and BPMES family members}

The structural diversity of BPSABATH and BPMES genes was analyzed through their exon/intron organization. The gene tree to analyze the interspecies relationship between $B p S A$ $B A T H$ and BPMES genes was constructed using the maximum likelihood method (Figure 2A). All BpSABATH genes contain introns; no genes without introns were observed (Figure 2B). Tandem duplicated pairs (BPSABATH1 with BPSABATH2 and BpSABATH5 and BpSABATH6 with BpSABATH7) showed similar gene structures. Every exon of the gene was similar to its tandemly duplicated sister gene and also showed a similar size (Figure 2B).

The BPMES gene structural analysis revealed that the number of exons varied from two to five. No genes lacking introns were observed, i.e., all genes contained introns (Figure 2B). Tandem duplicated gene pairs (BPMES1 through BpMES2, and BPMES6, BpMES7, BpMES8, BPMES9 through BpMES10) showed similar intron and exon structures, while the two tandem duplicates, BPMES5 and BpMES11 displayed a related intron/ exon structure.

The symmetric exons represent the same splicing phase at both ends and an excess of symmetric exons and phase 0 introns are expected to accelerate protein domain exchange, exon shuffling, and fusion in recombination (Gilbert 1987, Patthy 1987). According to the analyzed gene structures, the exons of six genes were symmetric with phase 0 introns and no exon was symmetric with phase 1 and 2 introns. Of the 37 introns of the ten BPSABATH genes, 20 were phase 0 , three were phase 1 and four were phase 2 (Figure 2B). Similarly, of the 31 introns of MES genes, 27 were phase 0 , two were phase 1 , and only two were phase 2 (Figure 2B).

The motif similarities and differences within BpSABATH genes were compared using the MEME online suite (Figure $2 C$ ). The BpSABATH gene family contains 11 distinct motifs (Figure $2 \mathrm{C}$; S Figure 1A). Overall, the most closely related members of the family showed a similar motif organization (BPSABATH5 and BpSABATH6 with BpSABATH7, and BpSABATH1 with BpSA$B A T H 2)$. Motifs 1 and 5 were shared by all the BpSABATHs, while 
motif 10 was present only in BPSABATH5 and 6. Further, motif 9 was specific to the BPSABATH5, 6, and 7. Motifs 2, 3, and 4 were shared among all BPSABATHs, except for BpSABATH1 and BpSA$B A T H 9$. The gene sequences of BpSABATH1, 2, 3, 8, and 10 lacked motifs $7,9,10$, and 11 which were mainly distributed in BpSABATH4, 5, 6, 7, and 9 presents in the N-or C-terminal. Similarly, the organizational variations of the motifs in BPMES were compared. Eleven different motifs were identified in the BPMES gene family and their logos were also extracted (S Figure 1B). The most closely related members within the family showed a similar motif organization (BPMES6 and BPMES7 with BpMES8, and BPMES5 with BpMES12). Motif 3 was shared by all BpMESs, except for BpMES11 which contained only one motif (motif 2) (Figure 2C), while motif 6 and 10 were present only in BpMES1 and 2. Also, motif 9 was specific to BpMES5, 7, 8, and 9, while motif 7 was shared among BPMES1, 2, and 3 (Figure 2C). The putative functions of the candidate genes are confirmed by the information about the gene ontology (Figure 2D).

\section{Multiple sequence alignment and homology modeling of BPSABATH and BPMES proteins}

The protein sequence alignment of BpSABATH proteins with CbSAMT showed the presence of SAM/SAH-binding residues as well as the aromatic moiety of the substrate (Figure 3 ). A total number of six residues that actively participate in the SAM/SAH were observed at 22-Ser, 57-Asp, 98-Asp, 99-ASP, 129-Ser, and 130-Phe. Likewise, 16 residues involved in SA binding were detected at 25-GIn, 145-Ser, 146-Ser, 147-Tyr, 148Ser, 149- Leu, 150-Met, and 151-Trp. Of the six SAM/SAH binding residues that have been studied in CDSAMT, BPSABATH4, BpSABATH5, BpSABATH6, BpSABATH7, BpSABATH8, and BpSABATH10 showed all six, while BPSABATH1 did not show any.

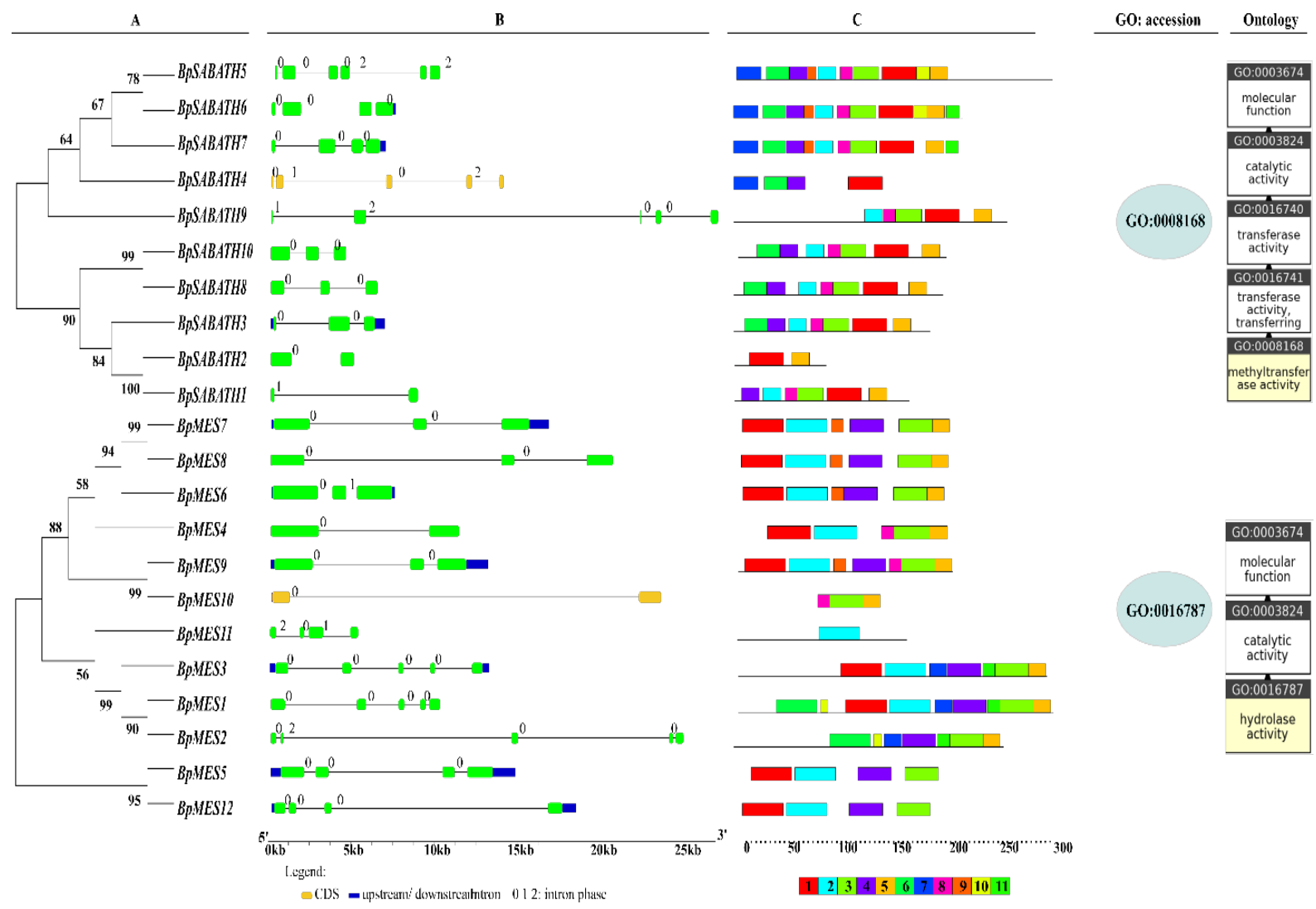

Figure 2

Intraspecies relationship, gene structure, motifs and gene ontology of SABATH and MES families: (A) The phylogenetic tree was constructed using the maximum likelihood method in MEGA X software with 1,000 bootstrap replicates (Kumar et al., 2018) to analyze the relationships between different BPSABATH and BPMES genes. (B) Structural features of the SABATH and MES genes in $B$. pendula. The coding regions (CDS) are indicated by green rectangles, while black lines between two exons represent the introns. Blue boxes indicate upstream/downstream UTRs. Intron phases are represented by the numbers above the intron (black line). Intron phases are likely to assist in exon shuffling, recombination fusion, and protein domain exchange (Gilbert, 1987, Patthy, 1987). (C) Schematic representation of the motifs in B. pendula SABATH and MES proteins. The lengths of the motifs can be estimated using the scale at the bottom of the figure. Observed elevan different motifs are listed at the bottom of the figure with different colors. (D) The information about the gene ontology is also shown to confirm the putative functions of the candidate genes. 
The presence of SA binding and aromatic residues still suggests the membership of the BpSABATH1 in the respective family. Further, BpSABATH2, BpSABATH3, and BpSABATH9 carried at least one of the SAM/SAH binding residues. The aromatic residue Val-311 was present in all the BPSABATH proteins except for BpSABATH1, BpSABATH2, BpSABATH3, and BpSABATH10, while BpSABATH2 only carried residue Tyr-147. BpSABATH9 contains the highest number of aromatic residues of all the BpSABATH and includes Tyr-147, Lue-210, Iso-225, and Phe-347, while residue Try-226 was present only in BpSABATH5, BpSABATH6, and BpSABATH7.
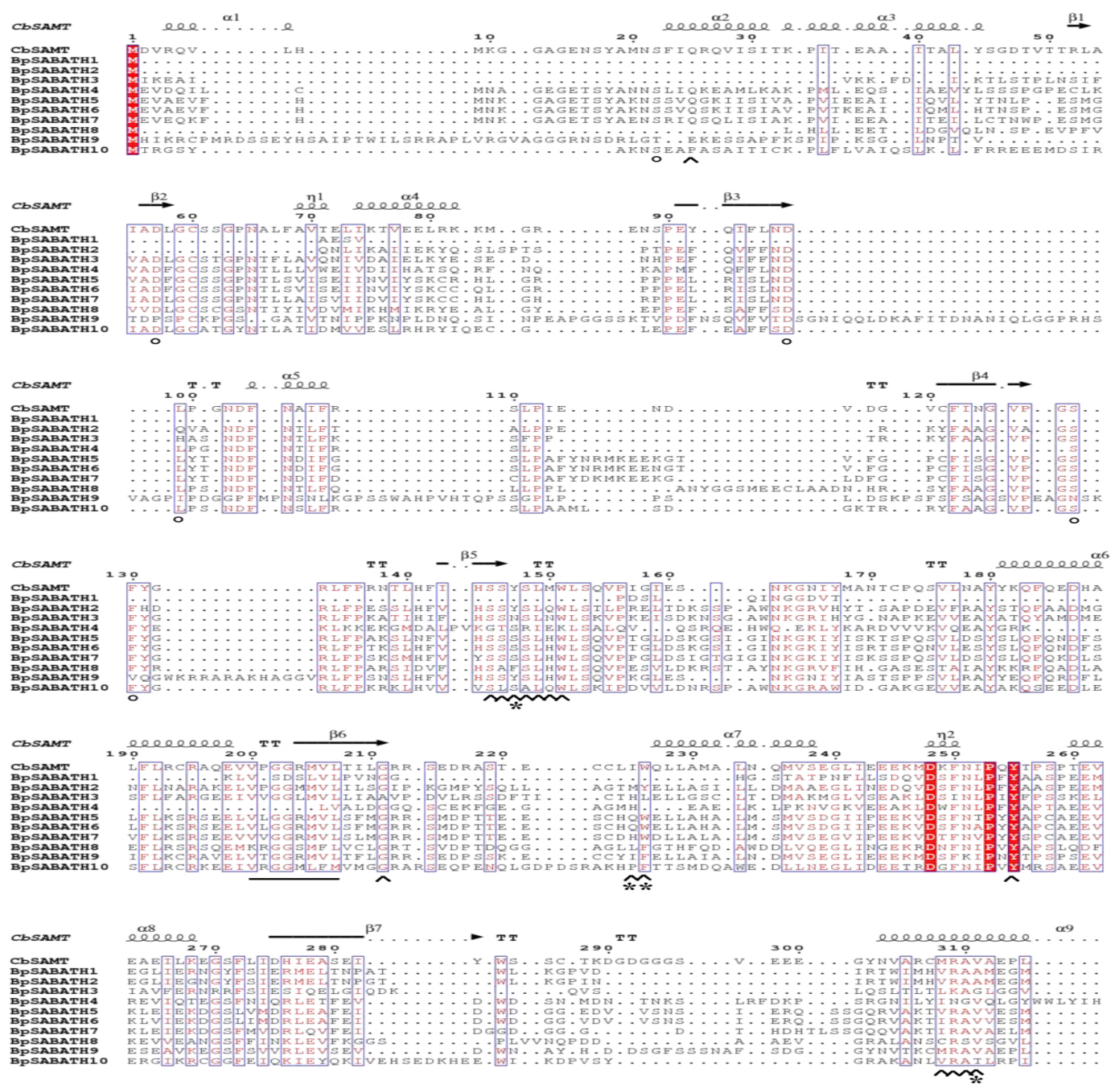

Figure 3

Multiple sequence alignment of BPSABATH family members: Structure-based multiple sequence alignment of $B$. pendula SABATH protein family members and, for comparison, CbSAMT from C. breweri (Ross et al., 1999). Blue frames indicate conserved residues, white characters in red boxes indicate strict identity, and red characters in boxes indicate amino acid sequence homology. The conserved domain of methyltransferase including the SAM-binding motif that had previously defined is highlighted with a black line (Joshi et al., 1998). The secondary structure elements above the alignment are those of the CbSAMT protein whose structure has been previously determined and described experimentally (Zubieta et al., 2003). The positions of residues involved in the SA substrate-binding and SAM/SAH-binding residues, identified by the three-dimensional structures, are indicated by black arrows and circles, respectively (Zubieta et al., 2003). Residues indicated by an asterisk are the aromatic moiety of the substrate and are important for substrate selectivity identified in a previous study (Zhao et al., 2008). The figure was prepared with the help of ESPript (Gouet et al., 1999). 
The protein sequence alignment of BpMES with NtSABP2 showed the presence of catalytic triad residues that were observed at 81-Ser, 210-Asp, and 238-His. A characteristic feature of the $\alpha / \beta$ hydrolase fold family is conserved in 8 of these enzymes (Figure 4). In the BPMES1 and BpMES3, the conserved Ser in the catalytic triad is replaced by Asp, a substitution previously found in active $\alpha / \beta$ hydrolases in animals (Holmquist 2000, Yang et al., 2008), while, in BpMES10 it is replaced by Met (Figure 4). Similarly, 14 residues that bind to SA were conserved at positions 13-Ala, 15-His, 81-Ser, 82-Leu, 107-Phe, 122-Tyr, 131-Trp, 136-Phe, 149-Met, 152-Phe, 155-Phe, 160-Leu, and 213-lle (Figure 4). Homology modeling of both gene families was conducted to analyze the protein structure similarities as well as to visualize the functional residues within the amino acid sequences identified in multiple sequence alignment ( $S$ Table 4).

\section{Evolutionary relationships and putative substrate specificities of BPSABATH and BPMES enzyme fami- ly members}

Phylogenetic clustering could preliminarily predict the functions of an unknown protein since grouped proteins in a clade showed similar gene structures and might possess similar functions (Kapteyn et al., 2007, Zhao et al., 2013). In addition, the proteins might be evolved from a recent common ancestor (Xie et al., 2014).
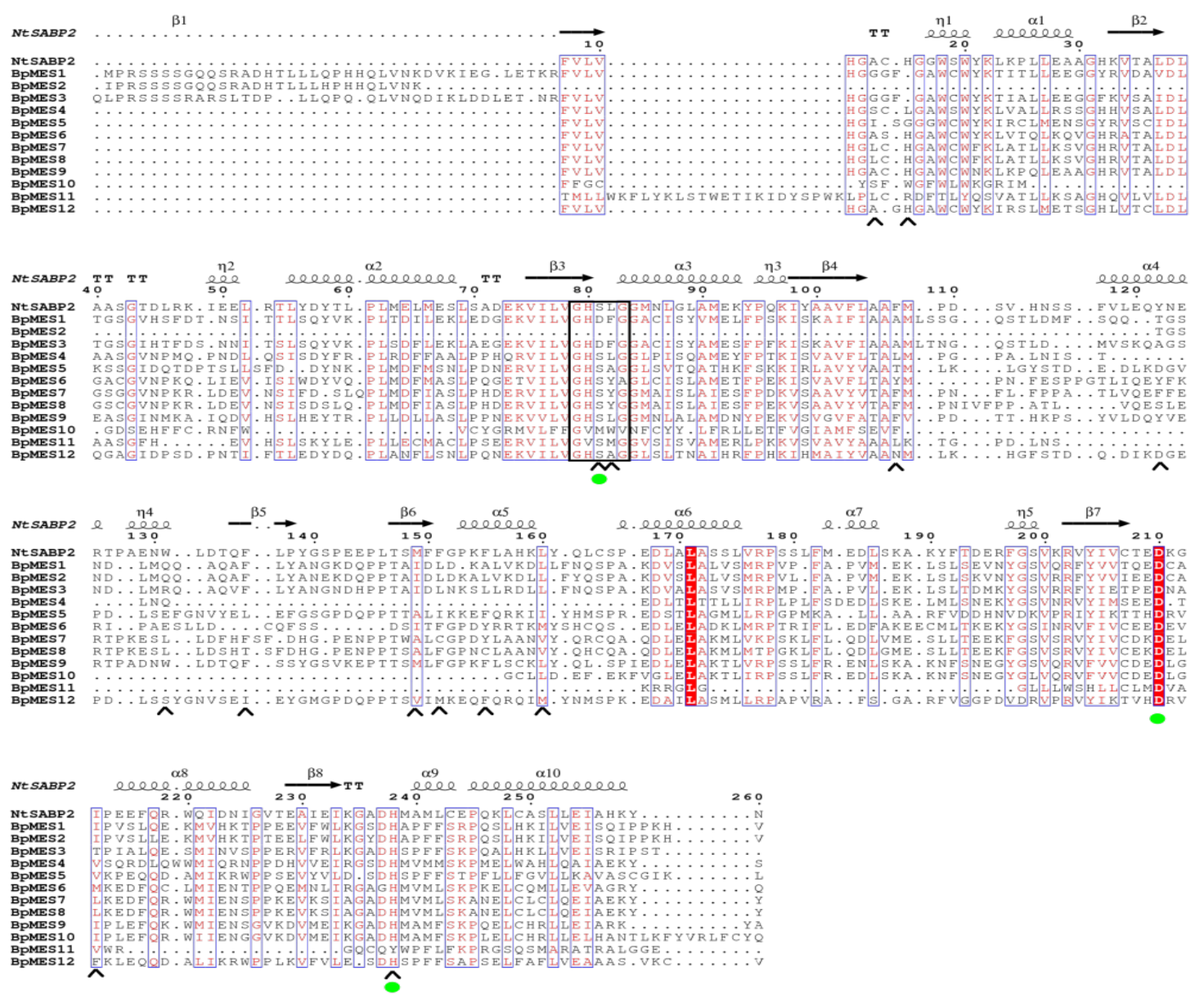

Figure 4

Multiple sequence alignment of BpMES family members: Multiple sequence alignment of $B$. pendula MES protein family members and, for comparison, NtSABP2 (Kumar et al., 2003). The blue frames represent the conserved residues, white characters in red boxes represent strict identity, and red characters in white boxes specify amino acid sequence homology. The lipase signature sequence of SABP2 is displayed with a black frame. The three conserved amino acids form a catalytic triad, S81, D210 and $\mathrm{H} 238$, commonly found in the hydrolase domain indicated by the green dot, conserved in BpMES (Kumar et al., 2003), while residues that contact to SA are indicated with an arrow (Forouhar et al., 2005). The figure was prepared with ESPript (Gouet et al., 1999). 


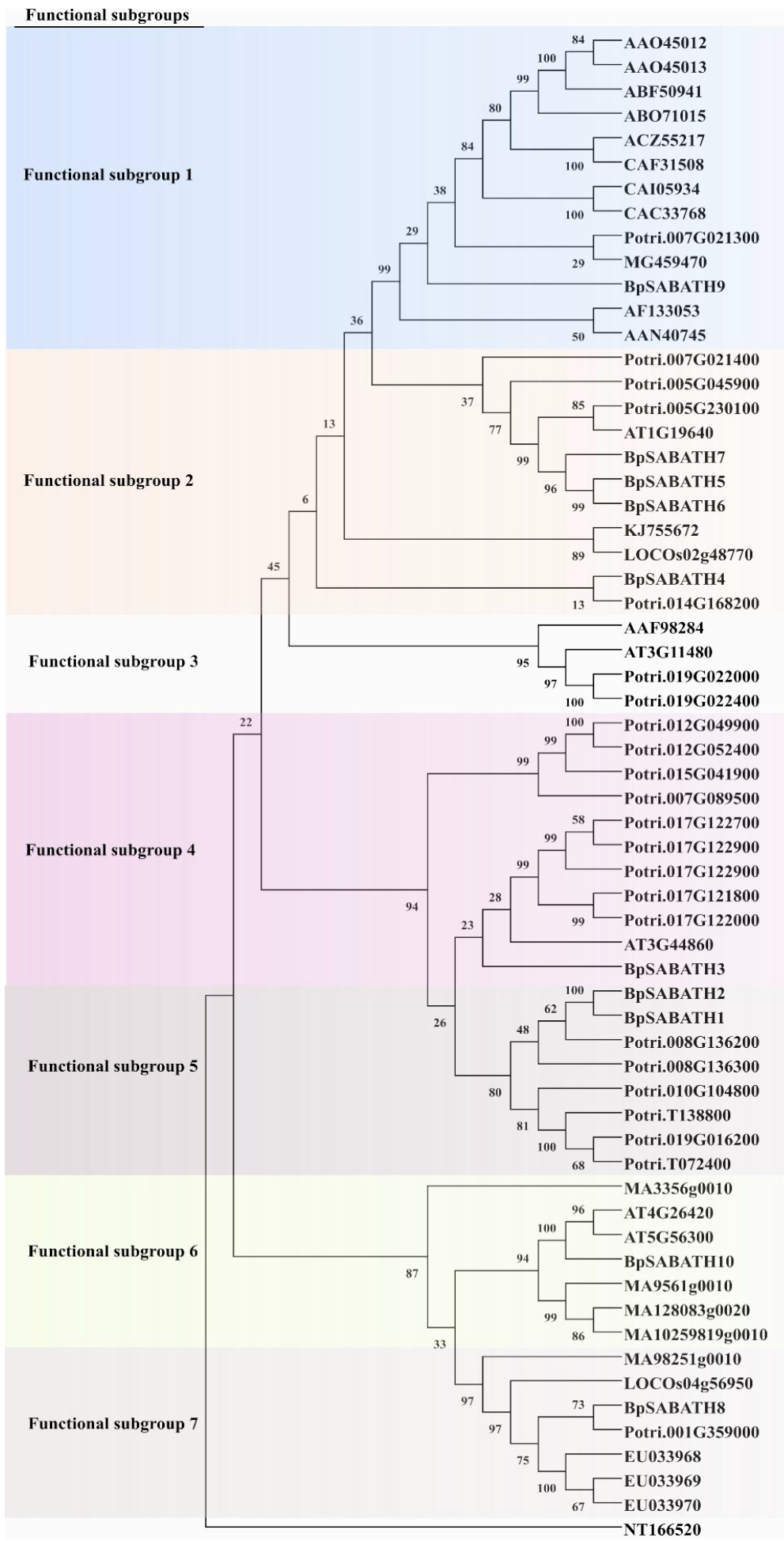

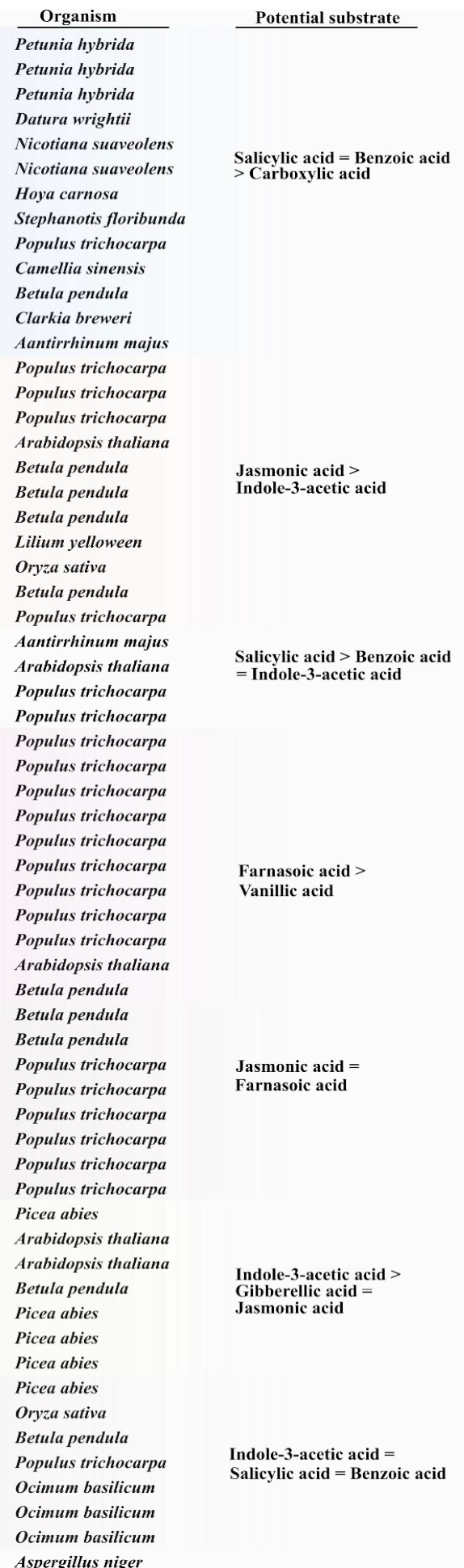

Figure 5

Potential substrates of BPSABATH protein family members according to phylogenetic clustering: Only functionally characterized protein sequences were used for the phylogenetic tree construction. The functional relationship between SABATH protein family members from the respective species was considered according to the subgroups formed in the gene tree. The gene tree was constructed using BpSABATH and 52 functionally characterized members of SABATH from other species (S. Table 1). A total number of 62 members of the SABATH family were used for the maximum likelihood gene tree in MEGA 7 software (Kumar et al., 2018). A member of the SABATH family, SAMT from Aspergillus niger (NT166520), was used as an outgroup. Numbers at nodes indicate bootstrap values calculated with 1,000 replicates. 
The probable role in plant and substrate specificity of BpSABATH proteins were determined based on a maximum likelihood gene tree (Figure 5) constructed using 10 BpSABATHs and 52 functionally characterized SABATHs from other plant species, including A. thaliana, P. trichocarpa, P. abies, and other respective species. Only functionally characterized SABATH members from the respective species were included to predict potential substrates of BpSABATH enzymes. The topology of the gene tree and distribution of the BpSABATHs, together with functionally characterized SABATHs (Figure 5), formed seven functional subgroups (functional subgroups 1 to 7).

Almost all functionally characterized SABATHs from functional subgroup 1 were observed to catalyze the conversion of SA and BA to SAMT and/or BSMT. Only one (BPSABATH9) of the $10 \mathrm{BpSABATHs}$ grouped with functional group 1. The resulting products are subsequently involved in various biological functions. Most of the members from functional subgroup 2 were involved in catalyzing JA and IAA and accommodate a maximum of four BpSABATHs, suggesting their potential functions. BpSABATH3, BpSABATH10, and BpSABATH8 were clustered in the functional subgroups 4, 6, and 7, respectively. Most of the functionally characterized members grouped in the functional subgroups 4, 6, and 7 showed higher enzymatic activity towards FA, JA, and IAA, respectively. Functional subgroups 3 and 5 did not include any members of the BpSABATHs, while functional group 7 included equal numbers of SABATHs that utilize IAA, SA, and BA as substrates.

In the gene tree, two groups of paralogous genes (BpSABATH1 and BpSABATH2; BPSABATH5, BPSABATH6, and BpSA$B A T H 7)$ were identified from the SABATH gene family in $B$. pendula (Figure 5). In addition, two pairs of orthologues, BpSABATH8 with Potri.001G359000 (highest enzymatic activity on indole-3-acetic acid; Han et al., 2017), and BpSABATH4 with Potri.014G168200 (no and lowest enzymatic activity on any tested substrates and indole-3-acetic acid, respectively; Han et al., 2017) were identified in the SABATH gene family in $B$. pendula that most probably have the same function. The clustering patterns of the gene tree provide a preliminary understanding for predicting the functions of an unknown protein since proteins grouped in one clade showed similar functions (Kapteyn et al., 2007, Zhao et al., 2013) and the proteins might have evolved from a recent common ancestor (Xie et al., 2014).

Various members of the MES gene family were identified and their functional characterization has been described in numerous plants (Lima Silva et al., 2019, Vlot et al., 2008, Yang et al., 2008, Zhao et al., 2009, Zhao et al., 2016). Here, the putative role and substrate specificity of $B$. pendula MES proteins were determined based on an ML gene tree (Figure 6) constructed using 23 functionally characterized MESs from other plant species, including A. thaliana, P. trichocarpa, and V. vinifera. Only $B$. pendula and functionally characterized MES protein members from the respective species were included to construct the gene tree and to predict the potential substrates of BpMES enzymes (Figure 6). The topology of the gene tree and distribution of $B$. pendula along with functionally characterized MESs formed four functional subgroups (functional subgroups
1 to 4). All the functionally characterized MES and SABP2 genes from functional subgroup 1 were observed to catalyze the conversion of MeSA to SA which is subsequently involved in various biological functions. Two (BpMES9 and BpMES10) of the 12 BPMES were included in functional subgroup 1. Functional subgroup 2 contained seven AtMES that showed hydrolyze activity towards MeSA, PNPA, and MelAA (Yang et al., 2008). In functional subgroup 3, all the functionally characterized MES members were involved in catalyzing MeJA and accommodated four BpMESs (BpMES4, BpMES6, BpMES7, and BpMES8), suggesting their potential functions.

B. pendula MES5, MES11, and MES12 formed a cluster in the functional subgroup 4. One of the functionally characterized AtMES17 (At3g10870) showed the highest and most specific activity towards MelAA, while AtMES17 (At4g16690) catalyzed MelAA as well as PNPA and MeJA (Yang et al., 2008). BPMES1, BpMES2, and BpMES3 were not clustered in any of the functional subgroups and gave no evidence about their putative function.

In the gene tree, three groups of paralogous genes (BPMES1, BpMES2, and BpMES3; BpMES7 and BpMES8; BpMES9 and $B P M E S 10$ ) were identified from the MES gene family in $B$. pendula (Figure 6). Also, two pairs of orthologues, BpMES5 with AtMES17 (enzymatic activity towards MelAA), and BpMES6 with At3g50440 (enzymatic activity towards MeJA) were identified in the MES gene family in B. pendula and most probably have the same function (Figure 6). 


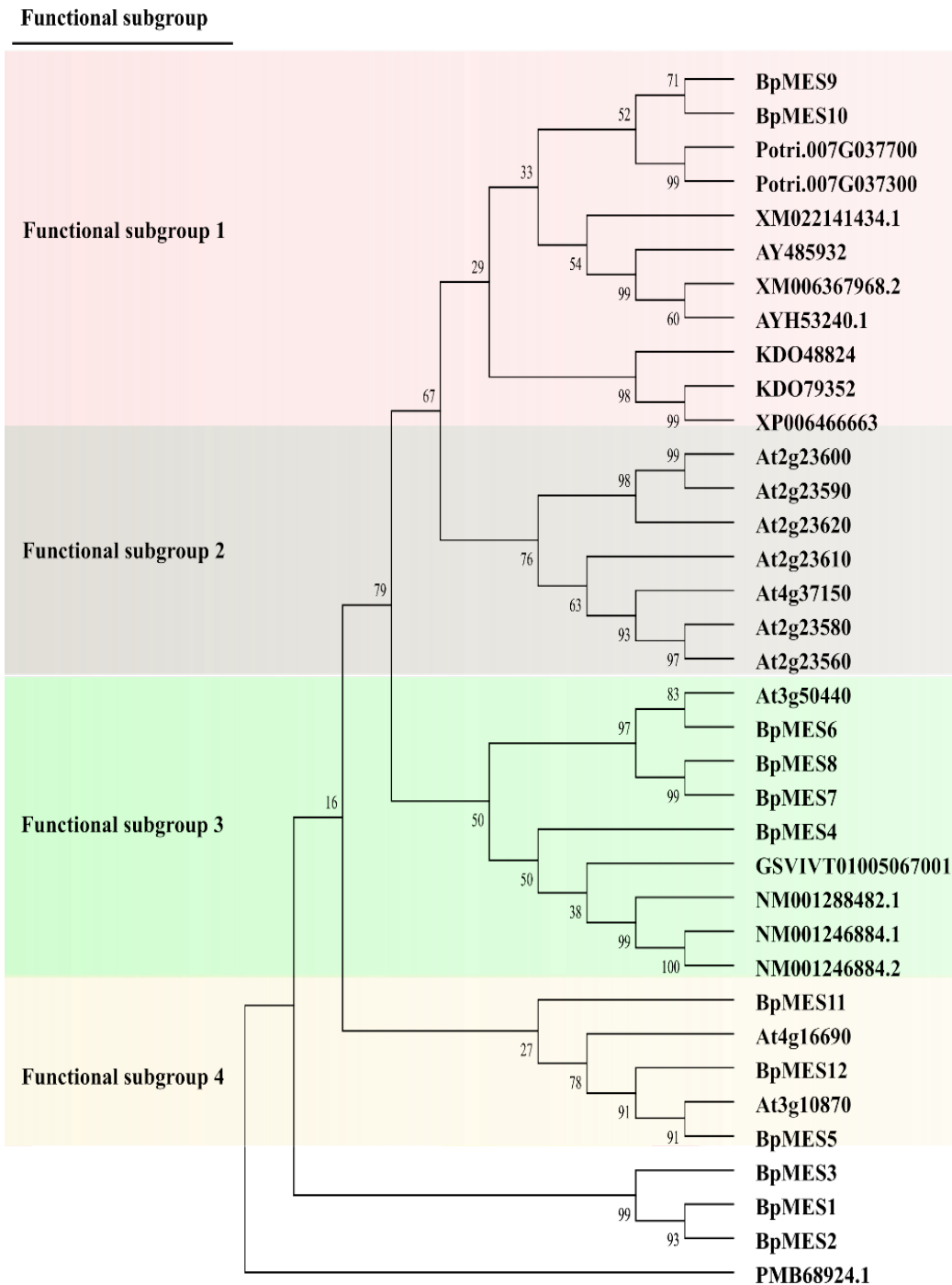

\begin{tabular}{|c|c|}
\hline Organism & Potential substrate \\
\hline $\begin{array}{l}\text { Betula pendula } \\
\text { Betula pendula } \\
\text { Populus trichocarpa } \\
\text { Populus trichocarpa } \\
\text { Helianthus annuus } \\
\text { Nicotiana tabacum } \\
\text { Solanum tuberosum } \\
\text { Lycium chinense } \\
\text { Citrus sinensis } \\
\text { Citrus sinensis }\end{array}$ & Methyl salicylate \\
\hline $\begin{array}{l}\text { Citrus sinensis } \\
\text { Arabidopsis thaliana } \\
\text { Arabidopsis thaliana } \\
\text { Arabidopsis thaliana } \\
\text { Arabidopsis thaliana } \\
\text { Arabidopsis thaliana } \\
\text { Arabidopsis thaliana } \\
\text { Arabidopsis thaliana }\end{array}$ & $\begin{array}{l}\text { Methyl salicylate > } \\
\text { Methyl indole-3-acetic acid }\end{array}$ \\
\hline $\begin{array}{l}\text { Arabidopsis thaliana } \\
\text { Betula pendula } \\
\text { Betula pendula } \\
\text { Betula pendula } \\
\text { Betula pendula } \\
\text { Vitis vinifera } \\
\text { Solanum tuberosum } \\
\text { Lycopersicon esculentum } \\
\text { Lycopersicon esculentum }\end{array}$ & Methyl jasmonate \\
\hline $\begin{array}{l}\text { Betula pendula } \\
\text { Arabidopsis thaliana } \\
\text { Betula pendula } \\
\text { Arabidopsis thaliana } \\
\text { Betula pendula }\end{array}$ & Methyl indole-3-acetic acid \\
\hline Betula pendula & \\
\hline $\begin{array}{l}\text { Betula pendula } \\
\text { Betula pendula }\end{array}$ & \\
\hline Beauveria bassiana & \\
\hline
\end{tabular}

Figure 6

Potential substrates of BpMES protein family members according to phylogenetic clustering: Functionally characterized protein sequences were only included for the phylogenetic tree construction. The functional relationship between MES protein family members from the respective species was considered according to the functional subgroups formed in the gene tree. The gene tree was constructed using BpMES and 24 functionally characterized members of SABATH from other species (S Table 1). A total number of 36 members of the MES family were used to construct the maximum likelihood gene tree in MEGA 7 software (Kumar et al., 2018). A member of the MES family from Beauveri abassiana (PMB68924.1) was used as an outgroup. Numbers at nodes indicate bootstrap values calculated with 1000 replicates.

\section{Promoter analysis of BPSABATH and BpMES gene family members}

Promoter regions of the BPSABATH and BPMES genes were retrieved from the available $B$. pendula genome (Salojärvi et al., 2017). Retrieved promoter sequences (S Table 5) were analyzed using the PlantCARE database to identify the putative cis-elements (S Table 6A and 6B). The presence of different cis-elements along with their frequencies in BPSABATH and BpMES gene promoter regions were evaluated (Figure 7 and 8). In total, 33 cis-acting elements were observed in the $10 \mathrm{BpSA}$ $B A T H$ genes. The TATA and CAAT box cis-elements were abundant and present in all the BpSABATH genes. The auxinresponsive element AuxRR was present in BpSABATH9, while the GC motif in BPSABATH1 and BpSABATH2. The MeJA responsive elements, the CGTCA, and TGACG motifs were present only in BpSABATH1, BpSABATH2, BpSABATH5, BpSABATH6, BpSA$B A T H 7$, and $B p S A B A T H 8$. Only one $S A$ responsive element, TCA, was present in BpSABATH1, BpSABATH2, BpSABATH3, BpSABATH4, BPSABATH9, and BpSABATH10.

In total, 66 different cis-acting elements were observed in the twelve $B$. pendula MES genes. The cis-elements, like the TATA and CAAT box, were considered abundant and were 


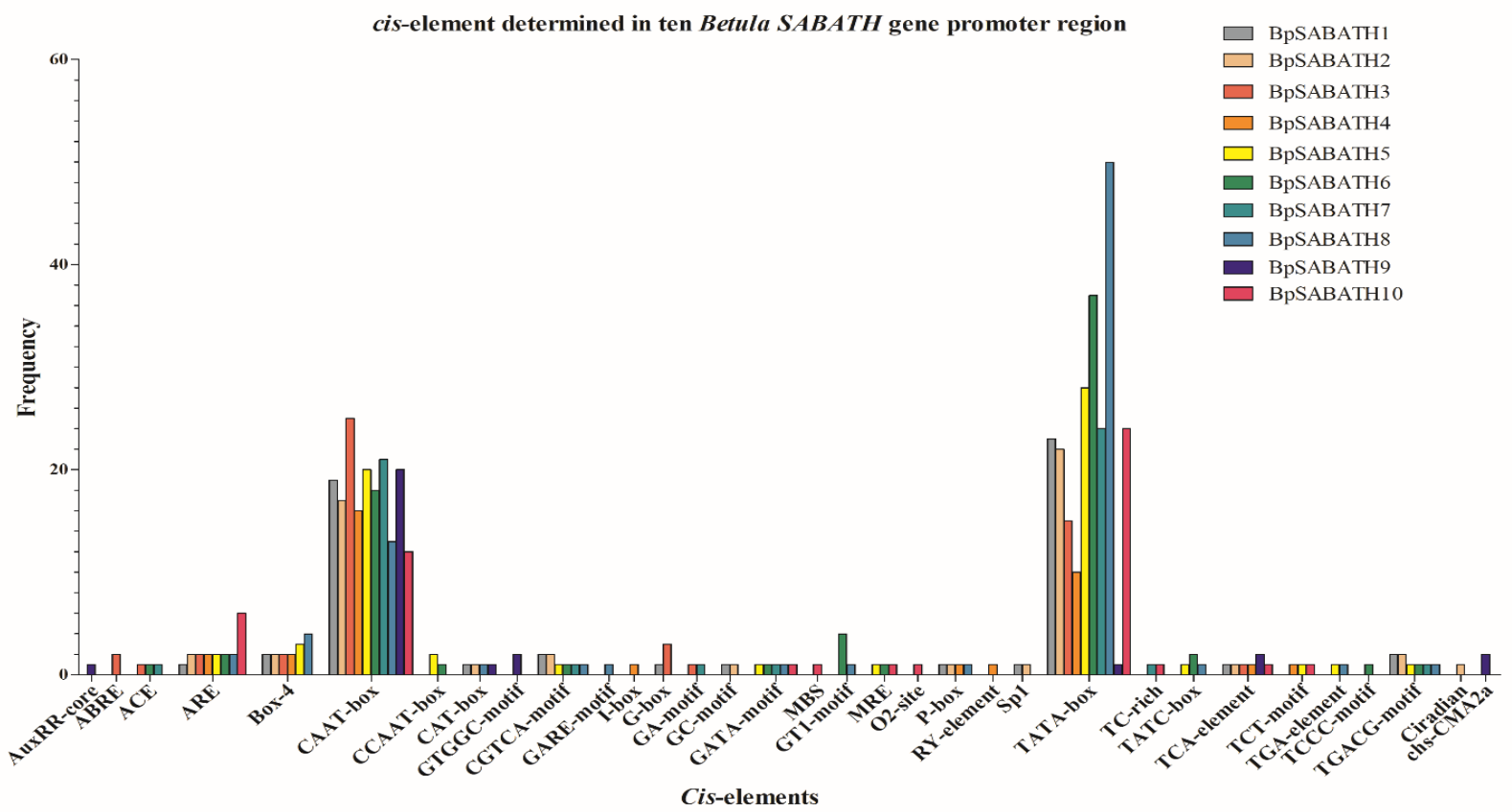

Figure 7

BpSABATH gene promoter analysis: Frequencies of identified cis-elements using the PlantCARE database (Lescot et al., 2002) in the promoter regions of $10 B P S A B A T H$ genes. Each BPSABATH is represented by a different color.

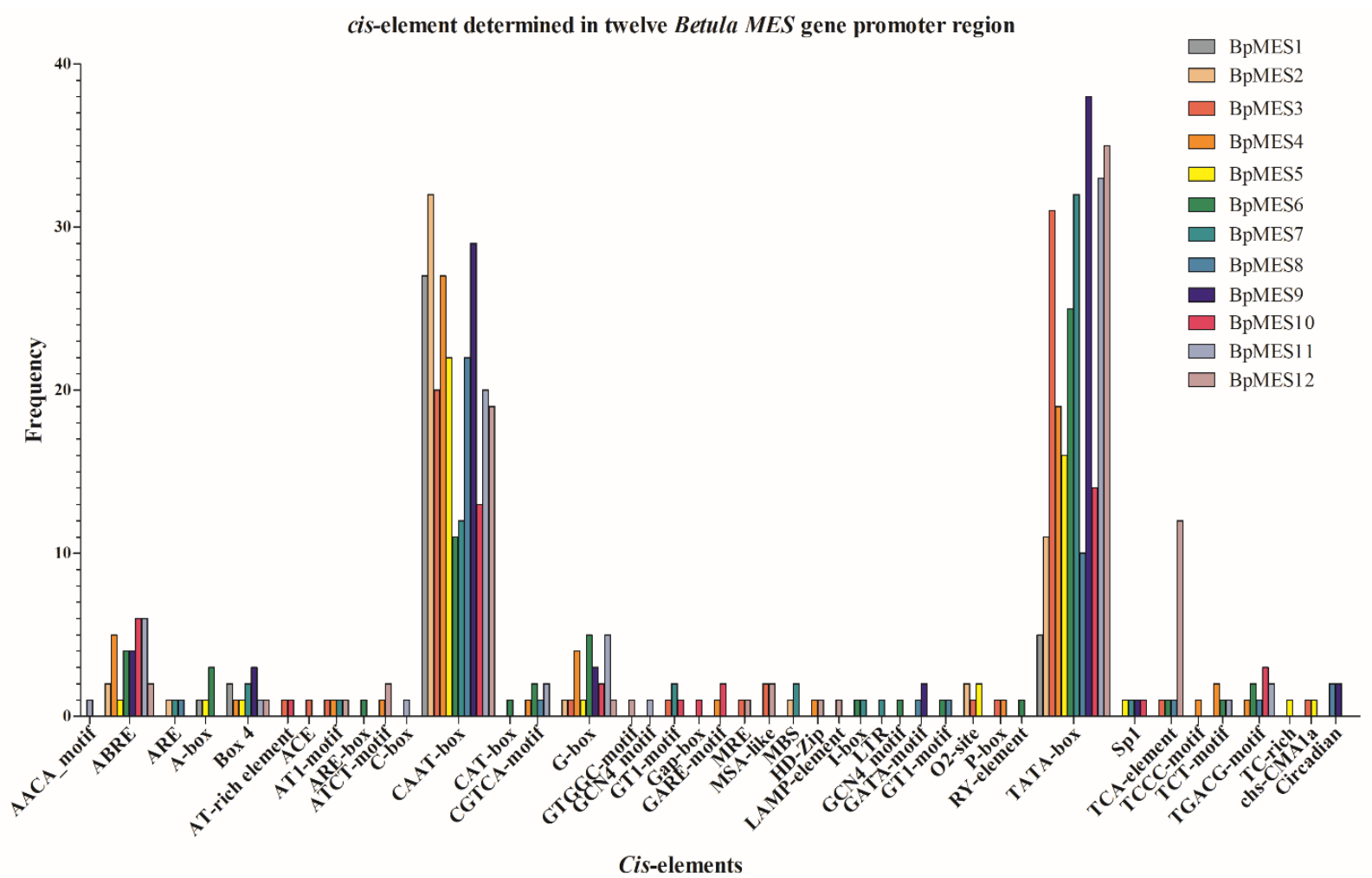

Figure 8

BPMES gene promoter analysis: Frequencies of identified cis-elements using the PlantCARE database (Lescot et al., 2002) in the promoter regions of 12 BpMES genes. Each BPMES is represented by a different color. 
present in all the Betula MES genes. The cis-acting elements, the AACA motif, $C$ box, and GCN4 motif, were present only on the promoter region of the BPMES11 gene, while ARE box, CAT box, GCN4 motif, and RY element were present only in the promoter region of the BPMES6 gene. Likewise, the GTGGC motif and LAMP elements were only present in the BPMES12 promoter regions. The ACE, GAP box, LTR, TCCC elements, and TC rich repeat elements were observed only in the promoter regions of BPMES3, BpMES10, BpMES7, BPMES4, and BPMES5 genes, respectively.

The MeJA responsive CGTCA motif was present on BpMES4, $B p M E S 6, B p M E S 8$, and BPMES11, and the TGACG motif was present in the BpMES4, BpMES6, BpMES8, BpMES10, and BpMES11 gene promoter regions, while the gibberellin-responsive GARE motif showed in BPMES4 and BPMES10, and P-box in BPMES3 and BpMES4 (S Table 6B). The abscisic acid-responsive ABRE was observed in BpMES2, BpMES 4, BPMES5, BPMES6, BpMES9, $B p M E S 10, B p M E S 11$, and BpMES12 promoters, while the TGA auxin responsible element was observed in BpMES1 and $B p M E S 9$. The TCA element involved in SA responses was present in BPMES3, BPMES6, BPMES8, and BPMES12, while TC-rich repeats involved in defense and stress responses were present only in BpMES5.

\section{Discussion}

Over the last few years, many plant genomes have been sequenced and this has contributed important information to plant improvement and development (Die et al., 2018). However, the genome sequencing of woody plant species is still rare e.g. Tuskan et al., 2006, Velasco et al., 2010, Nystedt et al., 2013, Salojärvi et al., 2017, Kuzmin et al., 2019, Chen et al., 2021. In this study, we used protein sequences of SABATH and MES and performed BLAST analyses against the $B$. pendula genome to extract candidate genes belonging to both families (Salojärvi et al., 2017).

To the best of our knowledge, following $A$. thaliana and $V$. vinifera, $B$. pendula is the third plant species, and the first tree species, in which the complete MES gene family has been recognized and comprehensively studied. Although the genome size of A. thaliana ( $135 \mathrm{Mbp})$ is, compared to V. vinifera $(\sim 500$ $\mathrm{Mbp}$ ) and B. pendula ( $440 \mathrm{Mbp})$, very small, it contains 20 members of the MES gene family (Yang et al., 2008), while $V$. vinifera and $B$. pendula only contain 15 and 12 members of MES, respectively (Zhao et al., 2016). Compared to the MES gene family, the SABATH gene family has been studied intensively in many plant species (Ament et al., 2010, Chaiprasongsuk et al., 2018, Chen et al., 2003, D'Auria et al., 2003, Han et al., 2017). The highest and lowest number of SABATH gene family members have been recorded in the $O$. sativa (41) and P. abies (10), respectively (Chaiprasongsuk et al., 2018, Zhao et al., 2008).

\section{Comparative bioinformatics analysis}

Plant SABATHs catalyze the methylation of numerous secondary metabolites, play an important role in different biological mechanisms, including stress responses, development, and growth (Ament et al., 2010, Chen et al., 2003, Effmert et al., 2005).

The $B$. pendula $10 \mathrm{BpSABATH}$ proteins were divided into two clades in the intraspecific SABATH members (Figure 2A). The BpMES proteins were divided into three distinct clades (Figure 2A). All the BpSABATH genes displayed a methyltransferase 7 domain (Methyltransf 7; S Table 7) and a conserved motif III that possessed SAM-binding sites (Joshi et al., 1998). The occurrence of motif III in 56 different plant species suggests it plays a major role in the binding of SAM-dependent O-methyltransferases to their specific substrate (Joshi et al., 1998, Vidgren et al., 1994). The crystallography analysis of the CbSAMT enzyme and the SA binding residues were also characterized in the BpSABATHs (Zubieta et al., 2003) (Figure 3). A total number of 3,5 , and 1 SAM/SAH binding residues were present in BpSABATH1, 2, and 9, while BpSABATH4-8 and 10 carried all six compared to the template CbSAMT amino acid sequence. The BpSABATH protein alignment also revealed the presence of aromatic-rich residues of the carboxyl bearing substrate-binding pockets that were previously observed in the detailed study of $A$. thaliana indole-3-acetic acid methyltransferase (AtIAMT) (Figure 3).

Except for BpMES2, all amino acid sequence alignments of BpMES enzyme family members displayed the presence of the catalytic $\alpha / \beta$ hydrolase domain ( $\alpha / \beta$ hydrolase; $S$ Table 7 ) conserved in the MES family, which is in agreement with $A$. thaliana MES enzymes (Yang et al., 2008). The signature amino acid sequence, conserved in the NtSABP2 proteins (Forouhar et al., 2005), was identified in all 12 BpMES members except for BpMES2 at positions 79-83 aa advocate their function (Figure 4). Except for BpMES1-2 and BpMES10-11, all the BpMES enzymes displayed the conserved catalytic triad found in the hydrolase domain identified through the protein profiling of NtSABP2 protein (Kumar et al., 2003). The conserved catalytic triad was also observed in $A$. thaliana and $V$. vinifera MES enzymes (Yang et al., 2008, Zhao et al., 2016). In the course of evolution, the mutation in the genomic sequence of a gene causes the amino acid substitution in a protein sequence, which results in the different protein structure, functional switch, or loss of function (Dagan et al., 2002, Zhang et al., 2003). Additionally, amino acid change creates a substrate switch in the enzyme families shows one of the characteristics of the mutations (Han et al., 2017). However, the presence of conserved domain, GO annotations and phylogenetic clustering guided us to predict the physiological function of the enzymes. We suggest, enzymatic and molecular experiments could be a better option to comment on the function of enzymes missing amino acid sequences. Moreover, the observed 14 residues in BpMES enzymes that bind to SA were consistent with the previous structural study of NtSABP2 (Forouhar et al., 2005). BpMES1, BpMES2, BpMES3, and BpMES11 carry some extra 25-43 amino acids at the $\mathrm{N}$ - terminal (Figure 4). The extended $\mathrm{N}$ terminal may not contain a directing signal peptide, suggesting that these BpMES enzymes are situated in the cytosol, similar to other members of the family (Yang et al., 2008). 


\section{Substrate specificities of BPSABATH and BPMES members}

Diverse SABATH and MES genes in numerous plants have been functionally described (Ament et al., 2010, Chaiprasongsuk et al., 2018, Chen et al., 2003, Han et al., 2017, Köllner et al., 2010). The members of a family from different species having similar functions will most probably be clustered together in the gene tree, suggesting the genes in the same clade might share a similar origin. It can be postulated that the SABATH and MES protein family members from different plant species with a higher similarity might have the same feature and function (Xie et al., 2014). Therefore, we can deduce the function of the new members of the SABATH and MES protein families in $B$. pendula according to their clustering pattern.

$\mathrm{SA}$ is one of the key molecules in plant species that are involved in plant development and many other mechanisms, including defense against various pathogens (Park et al., 2007, Ament et al., 2010, Köllner et al., 2010, Lima Silva et al., 2019). When SA is methylated by one of the SABATH enzymes to form $\mathrm{MeSA}$, the volatile ester, it functions as a signaling molecule after the plant experiences an infection (Park et al., 2007, Vlot et al., 2008, Zubieta et al., 2003). In P. trichocarpa, four SABATHs showed enzymatic activity towards SA, and PtSABATH4 displayed the highest activity towards SA (functional subgroup 1). Han et al. (2017) suggested that PtSABATH4 might be the only carrier of this salicylic acid carboxyl methyltransferase activity (Han et al., 2017). Notably, the SABATHs that utilized SA and/or BA above all other tested substrates formed one clade (Figure 5 , functional subgroup 1). The clustering is confirming the potential function of BPSABATH9, since it is the only member of the B. pendula clustered together with Potri.007G021300 (PtSABATH4). In A. thaliana, SABATH8 (At3g11480) is one of the members that converted SA to MeSA (Chen et al., 2003). It is unlikely that At3g11480 and the O. sativa SABATH3 (LOCs02g48770) could be cluster with the SABATHs that catalyze SAMT in other flowering plants (Figure 5). The uneven distribution across the gene tree of SABATH enzymes that methylate SA supports the occurrence of multiple independent evolutionary events (Chaiprasongsuk et al., 2018) and displays the paraphyletic status of SAMT genes in seed plants.

Studies have shown that A. thaliana IAMT (At5g55250) converts IAA into non-polar methyl-IAA (Qin et al., 2005). Of 28 PtSABATH enzymes in P. trichocarpa, six (PtSABATH2, 3, 12, 17, 21 , and 24) had very low enzymatic activity towards IAA, while PtSABATH1 showed a 40.5-fold higher activity (Han et al., 2017). One P. glauca (PgSABATH1) (Zhao et al., 2009) and the $P$. abies (PaSABATH1: PalAMT) (Chaiprasongsuk et al., 2018) enzyme showed the highest enzymatic activity towards IAA, grouped in the functional subgroup 7 (Figure 5). Similarly, among 23 O. sativa SABATHs, only one OsSABATH4 (LOCs04g56950) gene showed catalytic activity towards IAA (Zhao et al., 2008). Likewise, a single BpSABATH (BpSABATH8) enzyme accommodated in functional subgroup 7 (Figure 5), along with all the functionally characterized SABATHs from the different respective species which preferred IAA as a most favorable substrate and might actively take part in auxin homeostasis.
The homology modeling also suggested a structural similarity to the protein accession, 3b5i (Indole-3-acetic Acid Methyltransferase) (S. Table 4). Previous studies suggested that the IAMT genes in the P. abies, A. thaliana, O. sativa, P. trichocarpa, and B. pendula are conserved (Chaiprasongsuk et al., 2018, Qin et al., 2005, Zhao et al., 2008) and might have evolved from a common ancestor of seed plants. The presented analysis supports the conclusion that IAMT might be the earliest member of SABATH family since a monophyletic clade has formed by BpSABATH8 (functional subgroup 7) and different plant species, including $A$. thaliana, O. sativa, P. trichocarpa and P. glauca (Zhao et al., 2009), which is consistent with our study (Figure 5).

Several studies have concluded that MeJA is involved in many diverse mechanisms, including defense, flowering, and seed germination (Cheong et al., 2003). However, very little is known about the function of MeJA in woody plants, other than that it promotes the synthesis of traumatic resin ducts in $P$. abies (Martin et al., 2003). The biochemical analysis of SABATH in $P$. abies revealed that three enzymes select JA as a favorable substrate (Chaiprasongsuk et al., 2018), while in P. trichocarpa, nine SABATHs showed enzymatic activity towards JA (Han et al., 2017). The involvement of multiple SABATHs in preferring JA was consistent since multiple (four of ten) BpSABATHs clustered together with the $P$. trichocarpa JAMT (Figure 5). This observation is in contrast to $A$. thaliana, where only one member of AtSABATH (At1G19640) was identified as having catalytic activity towards JA (Seo et al., 2001). At1G19640 and PtSABATH3 displayed the highest level of enzymatic activity towards JA and clustered together with four BpSABATHs (BPSABATH4, BpSABATH5, BpSABATH6, and BpSABATH7), while distantly related to the three $P$. abies JAMTs (PaJAMT1, PaJAMT2, and PaJAMT3) clustered in a different clade (Figure 5). Although the three PaJAMTs are most similar to each other, they are the result of the latest gene duplication and displayed a divergence in biochemical properties that indicates a functional divergence after gene duplication (Chaiprasongsuk et al., 2018). BpSABATH1 and BpSABATH3 were clustered with six $P$. trichocarpa SABATHs preferring JA as well as FA as a substrate. It has been shown that members of the SABATH enzyme family utilize multiple substrates at different levels of enzymatic activity (Han et al., 2017), suggesting their multifunctional ability to survive under diverse stress conditions.

The esterase activities of fifteen $A$. thaliana MES enzymes with four methyl esters of phytohormones (IAA, SA, GA, and JA) were tested. Eleven $A$. thaliana MESs showed esterase activity with at least one of the three substrates IAA, SA, and JA, while no enzyme showed activity towards GA (Yang et al., 2008). The biochemical analysis suggests that the demethylation of methyl esters of phytohormones may play an important role in modulating the different biological functions (Westfall et al., 2013).

AtMES17 (At3g10870) was hypothesized to encode for MelAA esterase since amino acid sequences were firmly displayed vital residues and conserved domains. Additionally, it has been confirmed in vitro through biochemical analyses that AtMES17 encodes an esterase that specifically hydrolyzes Mel$A A$ to IAA, and it is presumed that this will also occur in vivo 
(Yang et al., 2008). The biochemical functional analysis suggests that AtMES17 is responsible for auxin homeostasis since MelAA could transport through membranes to neighboring cells where it could be hydrolyzed back to the active auxin IAA by one of the MES members. The gene tree showed the orthologous relationship between AtMES17 and BpMES5, since they formed a close clade, suggesting that both encode an enzyme with a similar function (Figure 6).

BpMES4, BpMES6, BpMES7, and BpMES8 were clustered together with functionally characterized MES enzymes (Figure 6) that more specifically hydrolyze MeJA than other tested substrates (Stuhlfelder et al., 2004, Zhao et al., 2016). The clustering of multiple BpMESs with LeMJE (NM001246884.1 and NM001246884.2) was similar to the VvMES study where three VvMES formed a clade with LeMJE (Zhao et al., 2016). In a previous analysis, evidence of an orthologous relationship between VvMES5 (GSVIVT01005067001: VvMJE1), LeMJE, and AtMJE (At3g50440) was not found, which is in contrast to this study and Zhao et al., 2006. However, all the enzymes preferring MeJA as a substrate grouped in a clade might be due to the involvement of many enzymes for different species. Here, BpMES6 formed a cluster with AtMJE, suggestingan orthologous relationship (Figure 6, functional subgroup 3). In the present study $V$. vinifera, S. lycopersicum and $A$. thaliana MJE and $B$. pendula genes including BpMES6 group in one clade. However, the bootstrap support of this clade is low (50\%). (Zhao et al., 2016).

VvMJE1 from grapevine utilizes MeJA as a substrate since it showed a very high esterase activity towards MeJA in biochemical analyses (Zhao et al., 2016). Considering the phylogenetic clustering of BpMES4, BpMES6, BpMES7, and BpMES8 with previously biochemically characterized members of the MES family, we can predict that these BpMES also hydrolyze MeJA esters. Further biochemical analysis will be required to confirm which of these shows significant enzymatic activity towards MeJA.

BpMES9 and BpMES10 were the only two members clustered together with two functional methyl esterase $S A B P 2$ genes from P. trichocarpa (Zhao et al., 2009) (Figure 6). Further, members of MES, including S. tuberosum, Lycium chinense, Helianthus annuus, and N. tobacum (Kumar et al., 2003) from functional group 1, specifically utilize MeSA as substrate. Interestingly, the two MeSA esterases from $P$. trichocarpa possess similar functions, however, a likely difference in biological roles was observed (Zhao et al., 2009). Comparatively, B. pendula have not been through intense evolutionary genome duplication events, possibly resulting in only one copy of SABP2 (Salojärvi et al., 2017, Zhao et al., 2009). The amino acid sequence of BpMES10 showed one mismatch at the conserved catalytic triad in the multiple sequence alignment, while BpMES9 carries all three conserved residues (Figure 4). Thus, we could speculate that BpMES9 is the most trusted candidate in catalyzing the demethylation of MeSA. The hypothesis also supports the previous study where BPMES9/BPSABP2 was comprehensively studied in different Betula species and was proposed as a candidate gene to revert MeSA to SA (Singewar et al., 2020a,
Singewar et al., 2020b). Additionally, in the process of surviving in the surrounding environment, plants undergo genomic alterations which could lead to the amino acid change, resulting in the substrate switch, protein structural change, or loss of function (Han et al., 2017). We suggest enzymatic substrate specificity experiments could enlighten the function of the putative enzyme that has a mismatch in the amino acid sequence.

Substrate specificity of MES has always been a point of curiosity that has led to the biochemical analysis of different MESs with various possible substrates (Kumar et al., 2003, Yang et al., 2008, Zhao et al., 2009, Zhao et al., 2016, Zhao et al., 2013). The study by Zhao et al., (2016) has shown that, in addition to MeJA, VvMJE1 also prefers MeSA, but only at a high concentration of the substrate (Zhao et al., 2016). Similarly, the MeSA esterase from both $N$. tabacum and $P$. trichocarpa showed the highest activity towards MeSA. The MeSA ester was also enzymatically active towards MelAA and MeJA at only very high concentrations of substrate, which was considered to be physiologically insignificant (Forouhar et al., 2005, Zhao et al., 2009). Further, the AtMES enzymes showed enzymatic activity towards multiple substrates with different affinity under the experimental setups, suggesting that possibly MES enzymes use more than one substrate (Yang et al., 2008). These initial studies encourage a detailed examination using diverse MES members from different species, which will be beneficial for recognizing the evolution behind substrate specificity among the MES plant family.

\section{Conclusions}

To the best of our knowledge, this is the first study that has attempted to gather information about SABATH and MES family members at gene and enzyme levels in B. pendula. The vital comparative bioinformatics analysis revealed discrete patterns present in the SABATH and MES family members, involved in the biosynthesis of many hormones, signaling molecules, and floral scent. The identification and extensive in silico analysis of the BpSABATH and BpMES genes and enzymes revealed their putative functions and substrate specificities. This vital information will be an asset for further studies on functional and enzymatic substrate specificity, respectively. Additionally, the advantageous information of candidate genes could be exploited for genetic modification or targeted mutagenesis and genotype building to decide the function of a gene. Further, trait-specific markers would be designed to breed birch varieties that are adapted to certain environments.

\section{Acknowledgments}

We are extremely grateful to PD Dr. Birgit Kersten for assisting with the data retrieval, to Dr. Niels Müller for consultation on 
the manuscript review (both Thünen-Institute of Forest Genetics, Grosshansdorf, Germany), and to all the staff of the Thünen-Institute of Forest Genetics, Grosshansdorf, Germany, for their support, including Katrin Groppe, Susanne Jelkmann, and Vivian Kuhlenkamp for technical assistance, and Dr. Hilke Schröder and Dr. Hans Hönicka for helpful discussions on the methylation and demethylation process, and Thanks are also to Ana Montalvao and Avneesh Kumar (Plant Breeding Institute, University of Kiel, Kiel, Germany) for assistance in R programing language. Many thanks to the staff from the Institute for Agricultural Process Engineering, Christian-Albrechts University of Kiel, Germany. Thanks are due to Nicola Wilton (Language Boutique", Hamburg, Germany) and Dina Führmann (Thünen Institute, Braunschweig, Germany) for English language editing.

\section{Data Availability}

The data used to support the findings of this study are available by the authors (kiran.singewar@thuenen.de, matthias.fladung@thuenen.de) upon request.

\section{Conflicts of Interest}

All authors of the research article have no conflicts of interest to disclose.

\section{Funding Statement}

The project was funded by the "Energiewende und Umweltinnovationen" state program for the economy; Ministry for Energy Transition, Agriculture, Environment, Nature and Digitalization of Schleswig-Holstein, Germany. [Project number: LPW-L/1.2/24] Principal Investigator: Dr. Christian R. Moschner.

\section{Supplementary materials}

Supplementary material is provided with the manuscript.

\section{References}

Alvarez MV, Moreira MdR, Roura SI, Ayala-Zavala JF, González-Aguilar GA (2015) Using natural antimicrobials to enhance the safety and quality of fresh and processed fruits and vegetables: Types of antimicrobials. In: Handbook of Natural Antimicrobials for Food Safety and Quality. Taylor TM (ed) Oxford: Woodhead Publishing, pp 287-313.

https://doi.org/10.1016/b978-1-78242-034-7.00013-x
Ament K, Krasikov V, Allmann S, Rep M, Takken FLW, Schuurink RC (2010) Methyl salicylate production in tomato affects biotic interactions. The Plant Journal 62(1):124-134. https://dx.doi.org/10.1111/j.1365-313X.2010.04132.x

Araminienè V, Varnagiryte-Kabasinskiene I (2014) Research on birch species in Lithuania: A review study. In: Research for Rural Development 2. pp 50-56.

Ashburner K, McAllister HA, Hague J, Brown A, Williams P, Williams M, Rix M (2013) The Genus Betula: A Taxonomic Revision of Birches. Royal Botanic Gardens, London: Kew Publishing

Aspelmeier S, Leuschner C (2004) Genotypic variation in drought response of silver birch (Betula pendula): leaf water status and carbon gain. Tree Physiology 24(5):517-528. https://dx.doi.org/10.1093/treephys/24.5.517

Atkinson MD (1992) Betula pendula Roth (B. Verrucosa Ehrh.) and B. pubescens Ehrh. Journal of Ecology 80(4):837-870. https://dx.doi.org/10.2307/2260870

Chaiprasongsuk M, Zhang C, Qian P, Chen X, Li G, Trigiano RN, Guo H, Chen F (2018) Biochemical characterization in Norway spruce (Picea abies) of SABATH methyltransferases that methylate phytohormones. Phytochemistry 149:146-154. https://dx.doi.org/https://doi.org/10.1016/j.phytochem.2018.02.010

Chen F, D'Auria JC, Tholl D, Ross JR, Gershenzon J, Noel JP, Pichersky E (2003) An Arabidopsis thaliana gene for methylsalicylate biosynthesis, identified by a biochemical genomics approach, has a role in defense. The Plant Journal 36(5):577-588. https://dx.doi.org/10.1046/j.1365-313X.2003.01902.x

Chen S, Wang Y, Yu L. et al. (2012) Genome sequence and evolution of Betula platyphylla Hortic Res 8, 37. https://doi.org/10.1038/s41438-021-00481-7

Cheong J-J, Choi YD (2003) Methyl jasmonate as a vital substance in plants. Trends in Genetics 19(7):409-413.

https://dx.doi.org/https://doi.org/10.1016/S0168-9525(03)00138-0

Dagan T, Talmor Y, Graur D (2002) Ratios of radical to conservative amino acid replacement are affected by mutational and compositional factors and may not be indicative of positive darwinian selection. Molecular Biology and Evolution, Volume 19, Issue 7. Pages 1022-1025, https://doi.org/10.1093/oxfordjournals.molbev.a004161

D'Auria JC, Chen F, Pichersky E (2003) Chapter eleven The SABATH family of MTS in Arabidopsis thaliana and other plant species. In: Recent Advances in Phytochemistry. Romeo JT (ed): Elsevier, pp 253-283. https://doi.org/10.1016/s0079-9920(03)80026-6

Delker C, Raschke M, Fau-Quint A, Quint M (2008) Auxin dynamics: the dazzling complexity of a small molecule's message. Planta 227, 929-941. https://doi.org/10.1007/s00425-008-0710-8.

Die JV, Gil J, Millan T (2018) Genome-wide identification of the auxin response factor gene family in Cicer arietinum. BMC Genomics 19(1):301. https://dx.doi.org/10.1186/s12864-018-4695-9

Dogru E, Warzecha H, Seibel F, Haebel S, Lottspeich F, Stöckigt J (2000) The gene encoding polyneuridine aldehyde esterase of monoterpenoid indole alkaloid biosynthesis in plants is an ortholog of the alpha/betahydrolase super family. 267(5):1397-406. https://doi.org/10.1046/j.1432-1327.2000.01136.x

Dubois H, Verkasalo E, Claessens H (2020) Potential of Birch (Betula pendula Roth and B. pubescens Ehrh.) for forestry and forest-based industry sector within the changing climatic and socio-economic context of Western Europe. Forests 11(3):336. https://dx.doi.org/http://dx.doi.org/10.3390/f11030336

Dudareva N, Murfitt LM, Mann CJ, Gorenstein N, Kolosova N, Kish CM, Bonham C, Wood K (2000) developmental regulation of methyl benzoate biosynthesis and emission in snapdragon Flowers. The Plant Cell 12(6):949. https://dx.doi.org/10.1105/tpc.12.6.949

Effmert U, Saschenbrecker S, Ross J, Negre F, Fraser CM, Noel JP, Dudareva N, Piechulla B (2005) Floral benzenoid carboxyl methyltransferases: From in vitro to in planta function. Phytochemistry 66(11):1211-1230. https://dx.doi.org/https://doi.org/10.1016/j.phytochem.2005.03.031

El-Gebali S, Mistry J, Bateman A, Eddy SR, Luciani A, Potter SC, Qureshi M et al. (2018) The Pfam protein families database in 2019. Nucleic Acids Res 47(D1):D427-D432. https://dx.doi.org/10.1093/nar/gky995

Fischer A, Lindner M, Abs C, Lasch P (2002) Vegetation dynamics in central european forest ecosystems (near-natural as well as managed) after storm events. Folia Geobotanica 37(1):17-32. https://dx.doi.org/10.1007/BF02803188

Forouhar F, Lee IS, Vujcic J, Vujcic S, Shen J, Vorobiev SM, Xiao R, Acton TB, Montelione GT, Porter CW, Tong L (2005) Structural and functional evidence for 
Bacillus subtilis PaiA as a novel N1-spermidine/spermine acetyltransferase. J Biol Chem 280(48):40328-40336. https://dx.doi.org/10.1074/jbc.M505332200

Gang H, Li R, Zhao Y, Liu G, Chen S, Jiang J (2019) Loss of GLK1 transcription factor function reveals new insights in chlorophyll biosynthesis and chloroplast development. Journal of Experimental Botany 70(12):3125-3138. https://dx.doi.org/10.1093/jxb/erz128

Gel B, Serra E (2017) karyoploteR: an R/Bioconductor package to plot customizable linear genomes displaying arbitrary data. https://dx.doi.org/10.1101/122838

Gilbert W (1987) The exon theory of genes. 52:901-5. https://doi.org/10.1101/sqb.1987.052.01.098

Han X-M, Yang Q, Liu Y-J, Yang Z-L, Wang X-R, Zeng Q-Y, Yang H-L (2017) Evolution and function of the populus SABATH family reveal that a single amino acid change results in a substrate switch. Plant and Cell Physiology 59(2):392-403. https://dx.doi.org/10.1093/pcp/pcx198

Hemery GE, Clark JR, Aldinger E, Claessens H, Malvolti ME, O'Connor E, Raftoyannis Y, Savill PS, Brus R (2010) Growing scattered broadleaved tree species in Europe in a changing climate: a review of risks and opportunities. Forestry: An International Journal of Forest Research 83(1):65-81. https://dx.doi.org/10.1093/forestry/cpp034

Holmquist M (2000) Alpha beta-hydrolase fold enzymes structures, functions and mechanisms. Current Protein and Peptide Science 1(2):209-235. https:// dx.doi.org/10.2174/1389203003381405

Hynynen J, Niemistö P, Viherä-Aarnio A, Brunner A, Hein S, Velling P (2009) Silviculture of birch (Betula pendula Roth and Betula pubescens Ehrh.) in northern Europe. Forestry: An International Journal of Forest Research 83(1):103119. https://dx.doi.org/10.1093/forestry/cpp035

Joshi CP, Chiang VL (1998) Conserved sequence motifs in plant S-adenosyl-L-methionine-dependent methyltransferases. Plant Molecular Biology 37(4):663-674. https://dx.doi.org/10.1023/A:1006035210889

Kapteyn J, Qualley AV, Xie Z, Fridman E, Dudareva N, Gang DR (2007) Evolution of Cinnamate/p-coumarate carboxyl methyltransferases and their role in the biosynthesis of methylcinnamate. The Plant cell 19(10):3212-3229. https://dx.doi.org/10.1105/tpc.107.054155

Köllner TG, Lenk C, Zhao N, Seidl-Adams I, Gershenzon J, Chen F, Degenhardt J (2010) Herbivore-induced SABATH methyltransferases of maize that methylate anthranilic acid using s-adenosyl-L-methionine. Plant Physiology 153(4):1795-1807. https://dx.doi.org/10.1104/pp.110.158360

Kong $\mathrm{H}$, Landherr LL, Frohlich MW, Leebens-Mack J, Ma H, DePamphilis CW (2007) Patterns of gene duplication in the plant SKP1 gene family in angiosperms: evidence for multiple mechanisms of rapid gene birth. The Plant Journal 50(5):873-885. https://dx.doi.org/10.1111/j.1365-313X.2007.03097.x

Koonin EV (2006) The origin of introns and their role in eukaryogenesis: a compromise solution to the introns-early versus introns-late debate? Biol Direct 1:22-22. https://dx.doi.org/10.1186/1745-6150-1-22

Koski V, Rousi M (2005) A review of the promises and constraints of breeding silver birch (Betula pendula Roth) in Finland. Forestry: An International Journa of Forest Research 78(2):187-198. https://dx.doi.org/10.1093/forestry/cpi017

Kumar D, Klessig DF (2003) High-affinity salicylic acid-binding protein 2 is required for plant innate immunity and has salicylic acid-stimulated lipase activity. Proceedings of the National Academy of Sciences 100, 16101-16106. https://dx.doi.org/doi.org/10.1073/pnas.0307162100

Kumar D, Klessig DF (2003) High-affinity salicylic acid-binding protein 2 is required for plant innate immunity and has salicylic acid-stimulated lipase activity. 100 (26) 16101-16106. https://doi.org/10.1073/pnas.0307162100

Kumar S, Stecher G, Li M, Knyaz C, Tamura K (2018) MEGA X: Molecular evolutionary genetics analysis across computing platforms. Molecular Biology and Evolution 35(6):1547-1549. https://dx.doi.org/10.1093/molbev/msy096

Kuzmin DA, Feranchuk SI, Sharov VV et al. (2019) Stepwise large genome assembly approach: a case of Siberian larch (Larix sibirica Ledeb). BMC Bioinformatics 20, 37. https://doi.org/10.1186/s12859-018-2570-y

Lescot M, Déhais P, Thijs G, Marchal K, Moreau Y, Van de Peer Y, Rouzé P, Rombauts $S$ (2002) PlantCARE, a database of plant cis-acting regulatory elements and a portal to tools for in silico analysis of promoter sequences. $\mathrm{Nu}$ cleic Acids Research 30(1):325-327. https://dx.doi.org/10.1093/nar/30.1.325
Lima Silva CCd, Shimo HM, de Felício R, Mercaldi GF, Rocco SA, Benedetti CE (2019) Structure-function relationship of a citrus salicylate methylesterase and role of salicylic acid in citrus canker resistance. Scientific Reports 9(1):3901. https://dx.doi.org/10.1038/s41598-019-40552-3

Martin DM, Gershenzon J, Bohlmann J (2003) Induction of volatile terpene biosynthesis and diurnal emission by methyl jasmonate in foliage of Norway Spruce. Plant Physiology 132(3):1586. https://dx.doi.org/10.1104/pp.103.021196

Moore RC, Purugganan MD (2003) The early stages of duplicate gene evolution. Proceedings of the National Academy of Sciences 100(26):15682. https://dx.doi.org/10.1073/pnas.2535513100

Nardini M, Dijkstra BW (1999) a/ $\beta$ Hydrolase fold enzymes: the family keeps growing. Current Opinion in Structural Biology 9(6):732-737. https://dx.doi.org/https://doi.org/10.1016/S0959-440X(99)00037-8

Nystedt B, Street N, Wetterbom A et al. (2013) The Norway spruce genome sequence and conifer genome evolution. Nature 497, 579-584. https://doi.org/10.1038/nature12211

Park S-W, Kaimoyo E, Kumar D, Mosher S, Klessig DF (2007) Methyl salicylate is a critical mobile signal for plant systemic acquired resistance. Science 318(5847):113. https://dx.doi.org/10.1126/science.1147113

Patthy L (1987) Intron-dependent evolution: Preferred types of exons and introns. FEBS Letters 214(1):1-7. https://dx.doi.org/10.1016/0014-5793(87)80002-9

Prévosto B, Curt T (2004) Dimensional relationships of naturally established European beech trees beneath Scots pine and Silver birch canopy. Forest Ecology and Management 194(1):335-348. https://doi.org/10.1016/j.foreco.2004.02.020

Qin G, Gu H, Zhao Y, Ma Z, Shi G, Yang Y, Pichersky E, Chen H, Liu M, Chen Z, Qu L-J (2005) An indole-3-acetic acid carboxyl methyltransferase regulates Ara bidopsis leaf development. The Plant Cell 17(10):2693-2704. https://dx.doi.org/10.1105/tpc.105.034959

Ranta H, Hokkanen T, Linkosalo T, Laukkanen L, Bondestam K, Oksanen A (2008) Male flowering of birch: Spatial synchronization, year-to-year variation and relation of catkin numbers and airborne pollen counts. Forest Ecology and Management 255(3):643-650. https://doi.org/10.1016/j.foreco.2007.09.040

Rosenvald K, Tullus A, Ostonen I, Uri V, Kupper P, Aosaar J, Varik M, Sõber J, Niglas A, Hansen R, Rohula G, Kukk M, Sõber A, Lõhmus K (2014) The effect of elevated air humidity on young silver birch and hybrid aspen biomass allocation and accumulation - Acclimation mechanisms and capacity. Forest Ecology and Management 330:252-260. https://doi.org/10.1016/j.foreco.2014.07.016

Ross JR, Nam KH, D'Auria JC, Pichersky E (1999) S-Adenosyl-I-Methionine: Salicylic acid carboxyl methyltransferase, an enzyme involved in floral scent production and plant defense, represents a new class of plant methyltransferases. Archives of Biochemistry and Biophysics 367(1):9-16. https://doi.org/10.1006/abbi.1999.1255

Salojärvi J, Smolander O-P, Nieminen K, Rajaraman S, Safronov O, Safdari P et al., (2017) Genome sequencing and population genomic analyses provide insights into the adaptive landscape of silver birch. Nature Genetics 49(6):904-912. https://dx.doi.org/10.1038/ng.3862

Seo HS, Song JT, Cheong JJ, Lee YH, Lee YW, Hwang I, Lee JS, Choi YD (2001) Jasmonic acid carboxyl methyltransferase: a key enzyme for jasmonate-regulated plant responses. Proc Natl Acad Sci U S A 98(8):4788-4793. https://dx.doi.org/10.1073/pnas.081557298

Singewar K, Moschner CR, Hartung E, Fladung M (2020a) Identification and analysis of key genes involved in methyl salicylate biosynthesis in different birch species. PLOS ONE 15(10):e0240246. https://dx.doi.org/10.1371/journal.pone.0240246

Singewar K, Moschner CR, Hartung E, Fladung M (2020b) Species determination and phylogenetic relationships of the genus Betula inferred from multiple chloroplast and nuclear regions reveal the high methyl salicylate-producing ability of the ancestor. Trees 34, 1131-1146. https://doi.org/10.1007/s00468-020-01984-x

Stuhlfelder C, Mueller MJ, Warzecha H (2004) Cloning and expression of a tomato CDNA encoding a methyl jasmonate cleaving esterase. European Journal of Biochemistry 271(14):2976-2983. https://dx.doi.org/10.1111/j.1432-1033.2004.04227.x 
Teale W, Paponov I, Palme K (2006) Auxin in action: signalling, transport and the control of plant growth and development. Nat Rev Mol Cell Biol 7, 847-859 (2006). https://doi.org/10.1038/nrm2020

Thompson JD, Higgins DG, Gibson TJ (1994) CLUSTAL W: improving the sensitivity of progressive multiple sequence alignment through sequence weighting, position-specific gap penalties and weight matrix choice. Nucleic acids research 22(22):4673-4680. https://dx.doi.org/10.1093/nar/22.22.4673

Tuskan GA, DiFazio S, Jansson S, Bohlmann J, Grigoriev I, Hellsten U, Putnam N et al. (2006) The Genome of Black Cottonwood, Populus trichocarpa (Torr. \& Gray). Science 313(5793):1596. https://dx.doi.org/10.1126/science.1128691

Velasco R, Zharkikh A, Affourtit J, Dhingra A, Cestaro A, Kalyanaraman A, Fontana $P$ et al. (2010) The genome of the domesticated apple (Malus $\times$ domestica Borkh.). Nature Genetics 42(10):833-839. https://dx.doi.org/10.1038/ng.654

Vidgren J, Svensson LA, Liljas A (1994) Crystal structure of catechol O-methyltransferase. Nature 368(6469):354-358. https://dx.doi.org/10.1038/368354a0

Vlot AC, Liu P-P, Cameron RK, Park S-W, Yang Y, Kumar D, Zhou F et al. (2008) Identification of likely orthologs of tobacco salicylic acid-binding protein 2 and their role in systemic acquired resistance in Arabidopsis thaliana. The Plant Journal 56(3):445-456. https://dx.doi.org/10.1111/j.1365-313X.2008.03618.x

Westfall CS, Muehler AM, Jez JM (2013) Enzyme action in the regulation of plant hormone responses. The Journal of biological chemistry 288(27):1930419311. https://dx.doi.org/10.1074/jbc.R113.475160

Xie R, Li Y, He S, Zheng Y, Yi S, Lv Q, Deng L (2014) Genome-wide analysis of citrus R2R3MYB genes and their spatiotemporal expression under stresses and hormone treatments. PLOS ONE 9(12):e113971. https://dx.doi.org/10.1371/journal.pone.0113971

Yang Y, Xu R, Ma C-J, Vlot AC, Klessig DF, Pichersky E (2008) Nactive methyl indole-3-acetic acid ester can be hydrolyzed and activated by several esterases belonging to the AtMES esterase family of Arabidopsis. Plant Physiology 147(3):1034.https://dx.doi.org/10.1104/pp.108.118224

Zhao N, Boyle B, Duval I, Ferrer J-L, Lin H, Seguin A, Mackay J, Chen F (2009) SABATH methyltransferases from white spruce (Picea glauca): gene cloning, functional characterization and structural analysis. Tree Physiology 29(7):947-957. https://dx.doi.org/10.1093/treephys/tpp023

Zhao N, Ferrer J-L, Ross J, Guan J, Yang Y, Pichersky E, Noel JP, Chen F (2008) Structural, biochemical, and phylogenetic analyses suggest that indole-3-acetic acid methyltransferase is an evolutionarily ancient member of the SABATH family. Plant Physiology 146(2):455. https://dx.doi.org/10.1104/pp.107.110049

Zhao N, Guan J, Forouhar F, Tschaplinski TJ, Cheng Z-M, Tong L, Chen F (2009) Two poplar methyl salicylate esterases display comparable biochemical properties but divergent expression patterns. Phytochemistry 70(1):32-39. https://doi.org/10.1016/j.phytochem.2008.11.014

Zhao N, Lin H, Lan S, Jia Q, Chen X, Guo H, Chen F (2016) VvMJE1 of the grapevine (Vitis vinifera) VvMES methylesterase family encodes for methyl jasmonate esterase and has a role in stress response. Plant Physiology and Biochemistry 102:125-132. https://doi.org/10.1016/j.plaphy.2016.02.027

Zhao N, Yao J, Chaiprasongsuk M, Li G, Guan J, Tschaplinski TJ, Guo H, Chen F (2013) Molecular and biochemical characterization of the jasmonic acid methyltransferase gene from black cottonwood (Populus trichocarpa). Phytochemistry 94:74-81. https://doi.org/10.1016/j.phytochem.2013.06.014

Zhang Y, Goritschnig S, Dong X, Li X (2003) A gain-of-function mutation in a plant disease resistance gene leads to constitutive activation of downstream signal transduction pathways in suppressor of npr1-1, constitutive 1 , The Plant Cell, Volume 15, Issue 11. Pages 2636-2646. https://doi.org/10.1105/tpc.015842

Zubieta C, Ross JR, Koscheski P, Yang Y, Pichersky E, Noel JP (2003) Structural basis for substrate recognition in the salicylic acid carboxyl methyltransferase family. The Plant Cell 15(8):1704. https://doi.org/10.1105/tpc.014548 